\title{
Complementary Actions of BDNF and Neurotrophin-3 on the Firing Patterns and Synaptic Composition of Motoneurons
}

\author{
María A. Davis-López de Carrizosa, Camilo J. Morado-Díaz, Juan J. Tena, Beatriz Benítez-Temiño, María L. Pecero, \\ Sara R. Morcuende, Rosa R. de la Cruz, and Angel M. Pastor \\ Departamento de Fisiología y Zoología, Facultad de Biología, Universidad de Sevilla, 41012 Sevilla, Spain
}

\begin{abstract}
Neurotrophins, as target-derived factors, are essential for neuronal survival during development, but during adulthood, their scope of actions widens to become also mediators of synaptic and morphological plasticity. Target disconnection by axotomy produces an initial synaptic stripping ensued by synaptic rearrangement upon target reinnervation. Using abducens motoneurons of the oculomotor system as a model for axotomy, we report that trophic support by brain-derived neurotrophic factor (BDNF), neurotrophin-3 (NT-3) or a mixture of both, delivered to the stump of severed axons, results in either the prevention of synaptic stripping when administered immediately after lesion or in a promotion of reinnervation of afferents to abducens motoneurons once synaptic stripping had occurred, in concert with the recovery of synaptic potentials evoked from the vestibular nerve. Synaptotrophic effects, however, were larger when both neurotrophins were applied together. The axotomy-induced reduction in firing sensitivities related to eye movements were also restored to normal values when BDNF and NT-3 were administered, but discharge characteristics recovered in a complementary manner when only one neurotrophin was used. This is the first report to show selective retrograde trophic dependence of circuit-driven firing properties in vivo indicating that NT-3 restored the phasic firing, whereas BDNF supported the tonic firing of motoneurons during eye movement performance. Therefore, our data report a link between the synaptotrophic actions of neurotrophins, retrogradely delivered, and the alterations of neuronal firing patterns during motor behaviors. These trophic actions could be responsible, in part, for synaptic rearrangements that alter circuit stability and synaptic balance during plastic events of the brain.
\end{abstract}

Key words: abducens motoneurons; synaptic plasticity; BDNF; NT-3; Trk receptors; axotomy; oculomotor; vestibular

\section{Introduction}

Neurotrophins provide part of the molecular background that promotes both synaptogenesis and stabilization of existing synapses in the adult brain, and therefore are crucial for plastic events such as regeneration or learning and memory (VicarioAbejón et al., 2002). These neurotrophin-mediated trophic effects, mostly studied in vitro, include the regulation of the number, composition and activity of synapses (McAllister et al., 1999; Mendell et al., 1999), as well as the regulation of firing patterns (Zhou et al., 2005; Youssoufian and Walmsley, 2007). Nonetheless, given the diversity of synaptic organization in the adult brain and the patterns of distribution of neurotrophins and receptors, an in vivo approach is required for ascertaining their specific roles under physiological conditions (Schinder and Poo, 2000; Caleo et al., 2003).

Lack of retrograde trophic support induced by axotomy in the

Received Nov. 4, 2008; revised Nov. 28, 2008; accepted Dec. 15, 2008

This work was supported by grants from Ministerio de Ciencia y Technologia-Fondo Europeo de Desarrollo Regional [MCYT-FEDER (BFI2006-08414)], Fundación de Investigación Médica Mutua Madrileña, Fundación Eugenio Rodríguez Pascual and Junta de Andalucía (P06-CTS-01420). M.A.D.-L.d.C. was scholar of the FISS (Fondo de Investigaciones Sanitarias de la Seguridad Social) in Spain. We thank Regeneron Pharmaceuticals for kindly providing BDNF and NT-3. We also thank Dr. Francisco Alvarez (Wright State University) for critical reading of this manuscript. We dedicate this paper to the memory of our colleague Prof. Francisco José Martínez-Guijarro.

Correspondence should be addressed to Dr. Angel M. Pastor, Departamento de Fisiología y Zoología, Facultad de Biología, Avda. Reina Mercedes, 6, 41012 Sevilla, Spain. E-mail: ampastor@us.es.

DOI:10.1523/JNEUROSCI.5312-08.2009

Copyright $\odot 2009$ Society for Neuroscience $\quad$ 0270-6474/09/290575-13\$15.00/0 adult CNS produces a profound synaptic stripping that decreases the motoneuronal responsiveness to afferent signals (Titmus and Faber, 1990). These alterations normally revert when target reinnervation is allowed to occur or when a new target is provided (Benítez-Temiño et al., 2005). The sequence of events for neurotrophins as target-derived factors is established. Neurotrophins are produced by muscle cells (Steljes et al., 1999; Omura et al., 2005) and then retrogradely and transsynaptically transported by motoneurons (Rind et al., 2005) that are endowed with neurotrophin receptors (Benítez-Temiño et al., 2004). Retrograde neurotrophin uptake and transport increases after nerve injury (Curtis et al., 1998), and neurotrophin signaling reaches the soma which influences cellular functions by different effectors and transcriptional pathways (Zweifel et al., 2005). The abducens motoneurons of the oculomotor system have firing patterns and afferent synaptic connectivity well correlated to the parameters of eye movement (Delgado-Garcia et al., 1986), and, thus, can be used to test whether firing and synaptic properties can be modulated by extrinsic trophic support after target-disconnection by means of axotomy.

If neurotrophins play a causal role, as target-derived factors, in regulating the discharge characteristics via alterations of the synaptic composition, manipulations that either decrease or increase neurotrophin supply should result in altered firing and neuronal sensitivities. Here, to test this prediction, we administered brain-derived neurotrophic factor (BDNF) and/or 
neurotrophin-3 (NT-3) to the stump of the severed axons of abducens motoneurons. Thus, we sought to challenge the synaptic stripping by two ways. We either prevented it by neurotrophin treatment starting at the moment of the lesion or promoted afferent reinnervation once the synaptic stripping had occurred. We also found a complementary role of BDNF and NT-3 in regulating the balance of tonic and phasic discharge properties of motoneurons, respectively, that could be causally related to the restoration of synaptic connectivity. This firing restoration could be prevented by the blockade of the neurotrophin receptor signaling with $\mathrm{K} 252 \mathrm{a}$, which resembles the effects of axotomy on discharge properties of motoneurons. Our data demonstrate that during synaptic remodelling, NT-3 and BDNF play trophic effects on synaptic inputs to motoneurons being NT-3 specific for phasic inputs and BDNF selective for tonic inputs.

\section{Materials and Methods}

Animals and surgical procedures. Experiments were conducted on adult cats weighing 2.0-2.5 kg obtained from authorized breeders (Universidad de Córdoba, Córdoba, Spain). Animals were either prepared for the chronic recording of eye movements and neuronal extracellular recordings in the abducens nucleus $(n=12)$ or those for histology were simply instrumented for the chronic delivery of neurotrophins $(n=10)$. Surgi$\mathrm{cal}$ and handling procedures for chronic and acute experiments followed the guidelines of the National Institutes of Health (http://oacu.od.nih. gov) and the specific recommendations for the preparation and maintenance of higher mammals during neuroscience experiments (NIH publication \#94-3207, 1994) and were in accordance with national legislation for the use and care of laboratory animals (R.D. 120/2005 BOE 252/ 34367-91, 2005).

Animals were surgically prepared for chronic recordings of eye movements and the electrical activity of abducens nucleus neurons. After a protective injection of atropine sulfate $(0.5 \mathrm{mg} / \mathrm{kg}$, i.m. $)$ to reduce vagal reflexes, the animal was anesthetized with sodium pentobarbital $(35 \mathrm{mg} /$ $\mathrm{kg}$, i.p.) and placed in a stereotaxic frame. Surgery was then performed under sterile conditions to implant stimulating electrodes, scleral coils, and the recording chamber. Two silver bipolar electrodes were implanted (supplemental Fig. 1, available at www.jneurosci.org as supplemental material) to stimulate the left VIth nerve at its exit from the brainstem and the right medial longitudinal fascicle (MLF) next to the oculomotor nucleus. Coils, made up of 2 turns of Teflon-insulated stainless-steel wire, were implanted in the sclera of both eyes. A square-shaped window $(5 \times 5 \mathrm{~mm})$ was drilled in the occipital bone to allow transcerebellar access to the brainstem with glass micropipettes during the recording sessions. A recording chamber made of acrylic resin was constructed around the window, which was sealed between recording sessions. Finally, a pedestal made of dental acrylic with embedded bolts was constructed to immobilize the head during the recordings. Postoperative care was provided to ensure the healthy state of the animal.

Chronic extracellular recordings. After $10-15 \mathrm{~d}$ of postoperative recovery, recording sessions started. The animal was restrained with elastic bandages, and placed in a Plexiglas box inside the scleral coil frame. Both the micromanipulator and the head-restraining device were located inside the coil frame. Eye movements were recorded by means of the magnetic field search-coil technique (Fuchs and Robinson, 1966). Eye coils were calibrated by rotating at known angles the magnetic field frame relative to the cat.

Extracellular recordings were performed with glass micropipettes beveled to a resistance of $1-2 \mathrm{M} \Omega$ and filled with $2 \mathrm{M} \mathrm{NaCl}$. The (left) abducens nucleus was approached stereotactically and located with the aid of the antidromic field potential produced by electrical stimulation of the VIth nerve. Abducens motoneurons were identified by their antidromic activation from the VIth nerve and by the collision test between the spontaneously occurring orthodromic and antidromically evoked action potentials. Abducens internuclear neurons were identified from the electrode implanted in the MLF. The extracellular neuronal activity was amplified and filtered at a bandwidth of $10 \mathrm{~Hz}-10 \mathrm{kHz}$ for display and digitalization purposes.

Data storage and analysis. Horizontal and vertical eye position of both eyes and neuronal activity were digitally stored for off-line analysis using a digitizing card (Power 1401, Cambridge Electronics Design). Computer programs were written in Matlab 6.5 to display the histogram of instantaneous firing frequency of the neuronal discharge (i.e., the reciprocal of the interval between two adjacent spikes) and the position of both eyes, and to select data between cursors. Relationships between neuronal firing rate (FR) (in spikes/s) and horizontal eye position (EP) (in degrees) were obtained by linear regression analysis to calculate the slope, i.e., the neuronal sensitivity to eye position ( $k$ ) (in spikes/s/degree), and the intercept $\left(F_{0}\right)$ (in spikes/s), i.e., the neuronal firing rate at straight-ahead gaze. Firing rate during fixations responded to the equation $\mathrm{FR}=k \cdot \mathrm{EP}+F_{0}$. Relationships between neuronal firing and eye velocity during spontaneous saccades were also obtained by linear regression analysis after subtraction of the position component $(k \cdot \mathrm{EP}) \mathrm{calcu}-$ lated from the previously known sensitivity to eye position. Thus, the equation used was $\mathrm{FR}-k \cdot \mathrm{EP}=r \cdot \mathrm{EV}+F_{0}$, where $r$ (in spikes/s/ degree/s) is the neuronal sensitivity to eye velocity (EV) (in degrees/s). Due to the impairment of eye movements of the affected eye, we used for computations the eye movement of the contralateral eye.

Synaptic potentials and peristimulus time histograms. Four animals were additionally implanted with silver bipolar electrodes in the vestibular nerve bilaterally (supplemental Fig. 1, available at www.jneurosci. org as supplemental material) during either the main surgical procedure or in a second surgical session. Single pulse electrical stimulation to the vestibular nerve with currents $<50 \mu \mathrm{A}$ produced typical extracellular field potentials in the abducens nucleus (Pastor et al., 1997) that were positive or negative-positive for the ipsilateral stimulation and mainly negative for stimulation applied to the contralateral vestibular nerve. One or more wavelets representing the afferent volley of activated presynaptic axons were typically seen at short latency after the stimulus artifact. To record extracellular field potentials, filters were set at DC-10 $\mathrm{KHz}$. For constructing the peristimulus time histograms (PSTH), we generated binned histograms (bin width $0.5 \mathrm{~ms}$ ) spanning $20 \mathrm{~ms}$ before and $50 \mathrm{~ms}$ after the stimulus for $200-400$ cycles of stimulation applied to either the contralateral or the ipsilateral vestibular nerves. The Schmidttrigger pulse obtained from action potentials was used to construct the PSTH and the stimulus artifact pooled at the time 0 bin, but excluded from computations. The intensity applied was $1.1 \times$ the current threshold necessary to evoke a detectable eye movement (Baker et al., 1981; Pastor et al., 1997). Neuronal responses were normalized to calculate the $z$-scores of a normal probability distribution to detect those bins that were statistically different from the mean of the response previous to the stimulus artifact. The contralateral nerve stimulation produced a facilitatory response within the $4-5 \mathrm{~ms}$ that followed the electrical stimulus onset. The ipsilateral stimulation produced a disfacilitation of the basal firing at the same latency. To quantify PSTH, the area above or below mean before the stimulus application was computed to compare responses among experimental groups.

Abducens nerve section and preparation of the implantable intraorbitary device. For the delivery of neurotrophins, we implanted the stump of the abducens nerve in a custom-made chamber to control the milieu that bathed it and to prevent axonal reinnervation of any orbital structure or other extraocular muscles (Delgado-García et al., 1988). The intraorbitary device consisted of a chamber that had two pieces of tubing inserted for fluid replacement and two electrodes as we recently described (DavisLópez de Carrizosa et al., 2008). The chamber was made with a standard polypropylene pipette tip shortened to $\sim 15 \mathrm{~mm}$ and thus having conical shape. The wider side of the cone had inserted the tip of two 6-cm long pieces of thin wall polytetrafluoroethylene (PTFE) (26 gauge; Small parts). The electrodes for electroneurographic recordings at the stump of the severed nerve were two PTFE insulated pieces, $6 \mathrm{~cm}$ long, $1 \mathrm{~mm}$ bare tip, made of multistranded stainless steel (located inside the chamber) and a single stranded silver wire ( $330 \mu \mathrm{m}$ outer diameter) located outside of the chamber that served as reference electrode (A-M Systems). The tubing and the inside electrode were glued in place to the wide side of the 
chamber. The narrow part of the conical chamber was left opened to $\sim 1$ $\mathrm{mm}$ wide to fit tightly the stump of the abducens nerve.

After a few control recording sessions to locate the abducens nucleus, the intraorbitary device was implanted under general anesthesia in a second surgical procedure. The orbit was approached temporally through a skin incision in the eyelid canthus at the left side. The abducens nerve was dissected free and the lateral rectus muscle removed. The distal end of the abducens nerve was inserted in the chamber by applying suction through the tubing system. The electrodes and tubing were passed under the skin and were affixed with dental acrylic to the head restraining system. A connection socket was used to solder the electrodes and a plastic chamber with a knuckled cap to protect the tubing endings. The total volume of the system was $\sim 10-15 \mu$ l including the dead space of the inlet and outlet tubing.

Administration of neurotrophins and inhibitor K252a. The protocol for neurotrophin delivery consisted of single injections of $15 \mu \mathrm{l}$ in the nerve chamber on alternate days during 4-6 weeks of a solution at a concentration of $15 \mu \mathrm{g} / \mu \mathrm{l}$ in saline. During the recording session, the chamber was rinsed with sterile saline and followed by the injection in the chamber of a dose of NT-3, BDNF or a mixture or both neurotrophins. The concentrations we used were in accordance with the doses used in studies in vivo (Mendell et al., 1999, Novikov et al., 2000). The selective inhibitor of the tyrosine protein kinase activity, K252a, was delivered at the concentration of $100 \mathrm{~nm}$ dissolved in saline with $2 \%$ dimethylsulfoxide and the mixture of both neurotrophins (Tapley et al., 1992). Finally, vehicle treatment consisted in the administration alone of sterile saline. Administration of neurotrophins either started the same day of axotomy [immediate administration protocol (IAP)] or delayed $15 \mathrm{~d}$ after the axotomy day [delayed administration protocol (DAP)].

Immunocytochemical procedures for fluorescent confocal microscopy. Animals were perfused intracardially with physiological saline followed by $4 \%$ phosphate buffer paraformaldehide. The brainstem at the abducens nucleus level was cut at $50-\mu \mathrm{m}$-thick coronal sections on a vibratome. The sections were washed in $0.01 \mathrm{~m}$ phosphate buffer, $\mathrm{pH}$ 7.4, with $0.9 \%$ saline and containing $0.1 \%$ Triton X-100 (PBS/TX) and then blocked with normal horse serum (1:10 in PBS/TX) and placed in different mixtures of primary antisera overnight at room temperature. Dual or triple color immunofluorescence for choline acetyltransferase (ChAT) (for motoneurons), synaptophysin (SYN) (for synaptic boutons) and glial fibrillary acidic protein (GFAP) (for astrocytes) was performed by mixing the following primary antibodies and dilutions: ChAT [goat polyclonal antibody (pAb); 1:300; Millipore], SYN (rabbit pAb; 1:500; Zymed Laboratories) and GFAP [mouse monoclonal antibody ( $\mathrm{mAb}$ ) $7 \mathrm{a} ; 1: 800$; Sigma]. To determine which fraction of the boutons was inhibitory, a series of experiments used the marker vesicular GABA/glycine transporter (VGAT) (Dumoulin et al., 1999; González-Forero et al., 2004), which is the vesicular $\gamma$-aminobutyric acid transporter also known as vesicular inhibitory amino acid transporter (VIAAT) that transports GABA and glycine into synaptic vesicles (rabbit pAb; 1:500, Millipore). Labeling of terminals with vesicular glutamate transporters 1 and 2 (VGLUT1 and VGLUT2) (guinea pig pAb; 1:300-1:1000; Millipore) yielded very low number of boutons on abducens motoneurons and no further attempts to characterize them were done. After several washes, the sections were incubated for $2-3 \mathrm{~h}$ in mixtures of secondary antibodies. Immunofluorescence was visualized with donkey anti-rabbit IgG coupled to fluorescein isothiocyanate (FITC) or Cy2, donkey anti-goat IgG coupled to FITC, tetramethylrhodamine isothiocyanate or Cy3 and donkey mouse IgG coupled Cy5 (The Jackson Laboratory). Secondary antibodies were diluted 1:50 in PBS/TX. Finally, sections were washed in PBS and mounted on glass slides and coversliped with Vectashield (Vector Laboratories).

Confocal microscopy (Leica; TCS SP2) at higher magnification $(\times 63$ oil immersion digitally zoomed at $\times 2$ ) was used to sequentially capture images at the same focal plane by means of different filters and later merged in Sigma scan or Image J (NIH). Gray scales were adjusted to expand the maximum dynamic range of the image. Neurons included in the quantitative analysis were randomly sampled at high magnification ( $\times 63)$ in 1-4 different focal planes separated by $2-3 \mu \mathrm{m}$ in the $z$-axis around the nuclear plane. Synaptophysin or VGAT boutons were counted to calculate the linear density of boutons. The synaptic coverage was also measured as the percentage ratio between the perimeter occupied by synaptophysin-inmunoreactive (IR), VGAT-IR terminals or GFAP-IR profiles over the ChAT-positive somata of motoneurons was also measured. Mean gray value as a measurement of the optical density of the section was also measured using square boxes of $30.4 \mu \mathrm{m}$ side ( 80 pixels side) sampled through the neuropil of images captured at $\times 40$. This measurement was considered as an index of the intensity of immunolabeling in the abducens nucleus and computed as the average gray value within the selection after background level subtraction. That is, the sum of the gray values of all the pixels (8-bit resolution) in the selection divided by the number of pixels. For comparisons between groups, oneway ANOVA at an overall level of significance of $p<0.05$ followed by post hoc comparisons were performed in SigmaStat version 3.1.

\section{Results}

Using the axotomy model of the well characterized abducens motoneurons, we tested the hypothesis that target-derived neurotrophic support modulates in adult motoneurons their synaptic composition and firing patterns. Axotomy leads to a profound reorganization of inputs to motoneurons that undergo stripping of synaptic boutons rendering these cells to a very plastic mode that helped us to test the synaptotrophic actions of neurotrophins.

Axotomy leads to firing alterations in abducens motoneurons Control abducens neurons maintained a tonic and regular discharge during eye fixations. Their firing rate increased monotonically for ocular fixations at successive higher angular positions directed toward the ipsilateral side of recording (the on-direction; leftward or temporal-directed for a left-side recorded motoneuron), and decreased or eventually ceased as the eye adopted more eccentric eye positions in the off-direction (Fig. $1 A$ ). The slope of the linear regression line between the firing rate and the eye position allowed us to calculate the neuronal sensitivity to eye position (measured in spikes/s/deg) (Fig. $1 \mathrm{D}$, circles). Motoneuronal activity was also related to eye velocity during saccades, thus motoneurons displayed high-frequency bursts during ondirected saccades or pauses preceding off-directed saccades (Fig. $1 A$ ). The slope of the linear regression analysis between firing rate and eye velocity was the sensitivity to eye velocity, namely $r$ (in spikes/s/deg/s).

The firing pattern of abducens motoneurons (Fig. $1 B$ ), but not internuclear neurons (Fig. 1C), was dramatically changed after lesion of the VIth nerve. Changes appeared gradually during the first week that followed lesion and were permanent and more noticeable throughout the second week and continued so during the entire course of the experiment (8-10 weeks). During the initial $15 \mathrm{~d}$ postlesion, axotomized motoneurons showed an overall reduction in firing rate. Motoneurons were accompanied also by a severe reduction of modulation related to eye position and velocity (Fig. $1 B$ ). Changes in the neuronal sensitivity to eye movement were noticed as reductions in the slopes between firing rate and both eye position and eye velocity (Fig. $1 D, E$, dots). Plotting the time course of changes in these two neuronal sensitivities ( $k$ and $r$ ) during axotomy revealed significant differences with control by 2 weeks after lesion (Fig. $1 F, G$ ). We used $15 \mathrm{~d}$ as a time border between two administration protocols of neurotrophins, because it is known that during these initial $15 \mathrm{~d}$, motoneurons undergo a profound synaptic stripping (our data, see below) (Delgado-Garcia et al., 1988). Thus, if neurotrophins were administered immediately after the axotomy, we hypothesized that synaptic stripping could be, at least partly, prevented, and we termed this procedure the IAP. To the contrary, if synaptic stripping was allowed to occur during the $15 \mathrm{~d}$ that ensued 
axotomy, the subsequent neurotrophin administration would in turn facilitate the afferent reinnervation during the so-called DAP.

Complementary firing restoration after single-neurotrophin administration Neurotrophin administration through the IOD directly into the nerve stump of the VIth nerve led to firing restoration in abducens motoneurons that could be appreciated as early as $48 \mathrm{~h}$ after treatment. Treatment with BDNF and NT-3 produced distinct changes in the firing pattern of motoneurons. Thus, while BDNF led to the restoration of the tonic firing (Fig. $2 B$ ), NT-3, in turn, restored the bursting behavior of motoneurons (Fig. 2C) (see Fig. $2 A$ for comparison with control), without altering in any case (during axotomy of the VIth nerve or during treatment with neurotrophins) the firing of abducens internuclear neurons (data not shown). Thus, BDNF and NT-3 produced complementary aspects of recovery in the firing of abducens motoneurons. BDNF treatment produced a significant recovery of the eye position sensitivity for both the immediate- and the delayedadministration protocols (Fig. 3A). To the contrary, BDNF did not produce any recovery in the eye velocity sensitivity (Fig. $3 B$ ) as bursts were of reduced amplitude (Fig. $2 B$ ). Sorting the firing rate-to-eye position plots in those fixations obtained after on-directed saccades versus those obtained after offdirected saccades yielded two eye position sensitivities, $k$-on and $k$-off. Comparison of the results obtained indicated that both recovered to control values after BDNF or BDNF+NT-3, but not after NT-3 treatment (not illustrated).

A mirror-image pattern occurred after NT-3 administration; in essence, the bursting behavior recovered but not the tonic firing of the motoneuron during spontaneous fixations (Fig. 2C). Quantification of the neuronal sensitivities for both the immediate and delayed administration protocols showed that the eye position sensitivity adopted an intermediate value, different from the axotomy value but different also from the control value (Fig. $3 A$ ). However, the eye velocity sensitivity showed a dramatic recovery reaching an average value higher than control (Fig. 3B) as motoneurons showed robust bursts during saccades (Fig. $2 C)$. This supranormal recovery of the eye velocity sensitivity resembled that found in the Ia-spinal motoneuron synaptic efficacy described by Mendell et al. (1999) when using NT-3 in axotomized and also in control motoneurons.

Plotting of the eye velocity versus eye

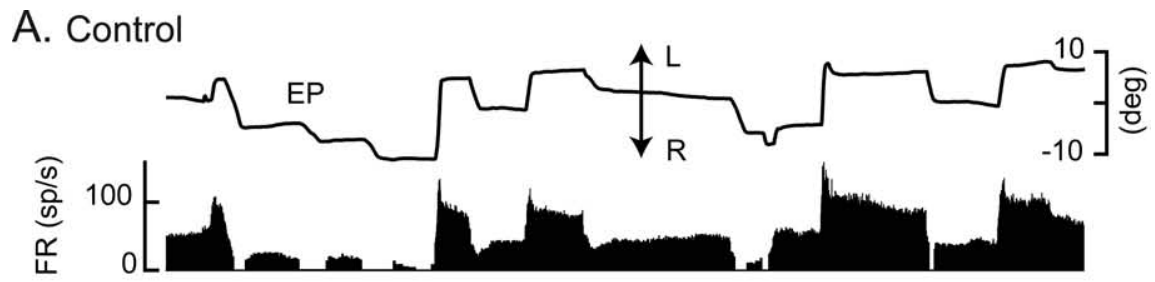

B. Axotomy

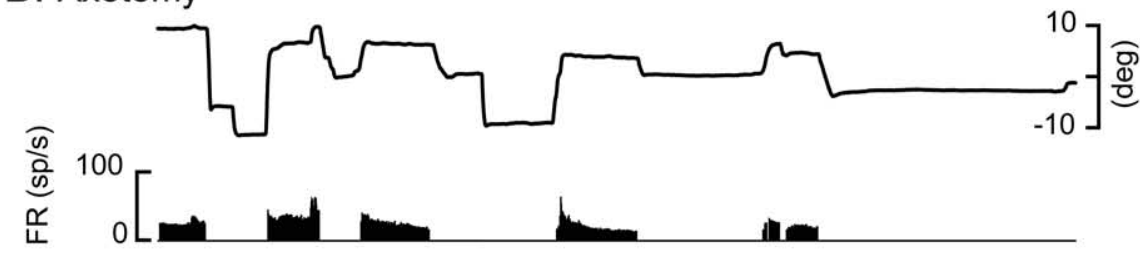

\section{Abducens internuclear neuron}
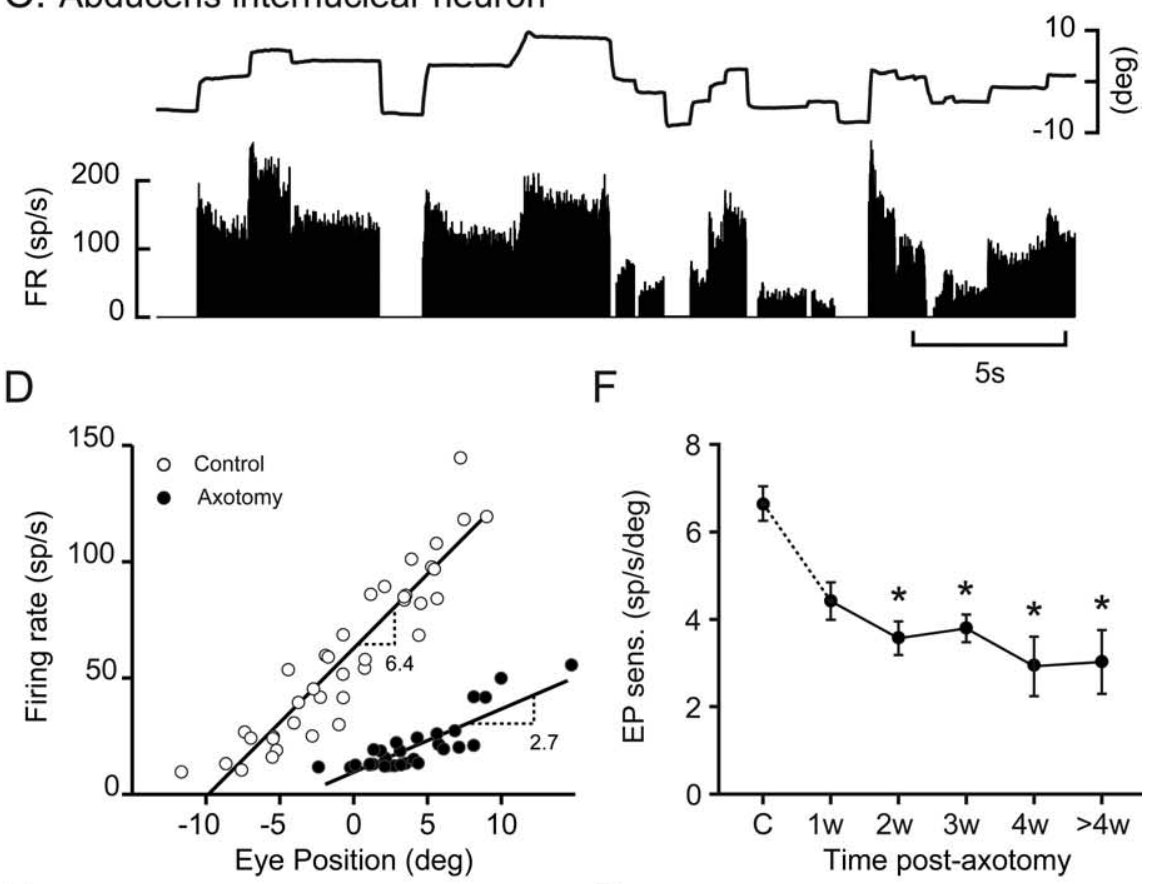

E

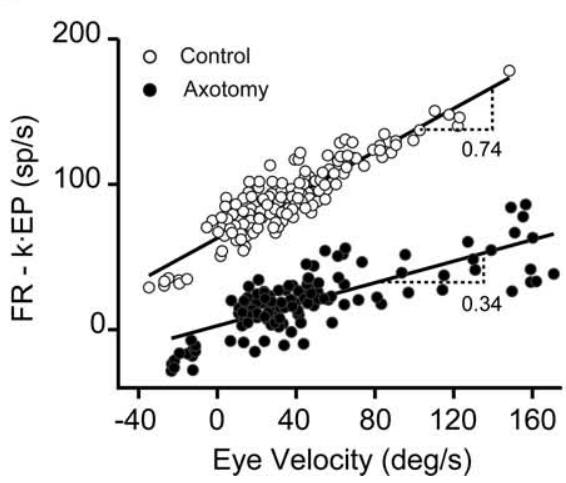

G

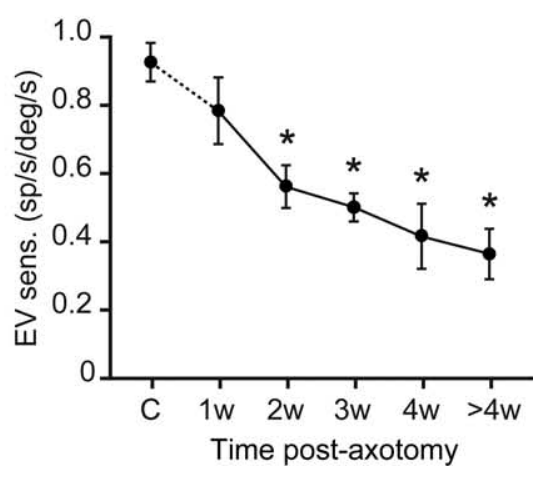

Figure 1. Effects of Vlth nerve axotomy on the discharge properties of abducens neurons with prevented regeneration and reinnervation of a target. $\boldsymbol{A}$, Firing profile of a control abducens motoneuron during spontaneous eye movements. $L$ and $R$ are left and right directions of movement, respectively. $\boldsymbol{B}$, Same as $\boldsymbol{A}$, but for a motoneuron $20 \mathrm{~d}$ after axotomy. Note reduced firing during both saccades and spontaneous fixations. $\boldsymbol{C}$, Abducens internuclear neurons always showed a normal firing pattern after lesion of the lateral rectus nerve. $\boldsymbol{D}$, Calculation of eye position sensitivity for the motoneurons shown in $\boldsymbol{A}$ and $\boldsymbol{B}$. The slopes of the regression lines are the neuronal sensitivity to spontaneous eye movements $(k$; in spikes $/ \mathrm{s} / \mathrm{deg})$. Linear relationships were $F R=$ $6.4 \mathrm{EP}+62.6$ for the control (circles; $r=0.92 ; p<0.001$ ), and FR $=2.7 \mathrm{EP}+1.0$ (dots; $r=0.9 ; p<0.001$ ) for the axotomized motoneuron. $\boldsymbol{E}$, Same as $\boldsymbol{D}$, but for eye velocity sensitivity. Linear relationships were $\mathrm{FR}^{\prime}=0.74 \mathrm{EP}$ (Figure legend continues.) 
A. Control

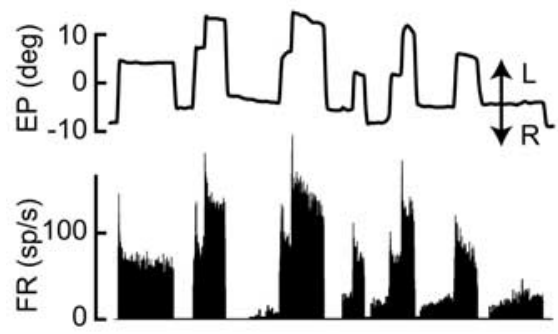

B. BDNF
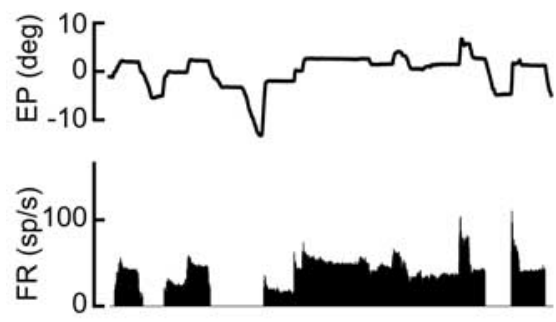

C. NT-3

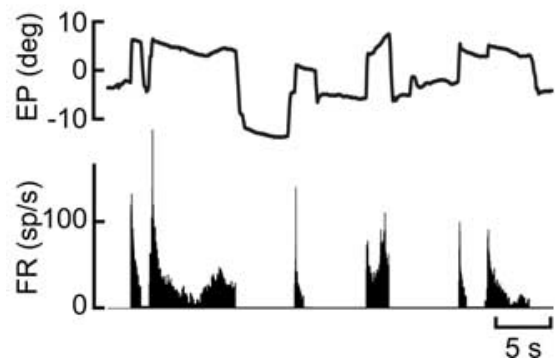

D

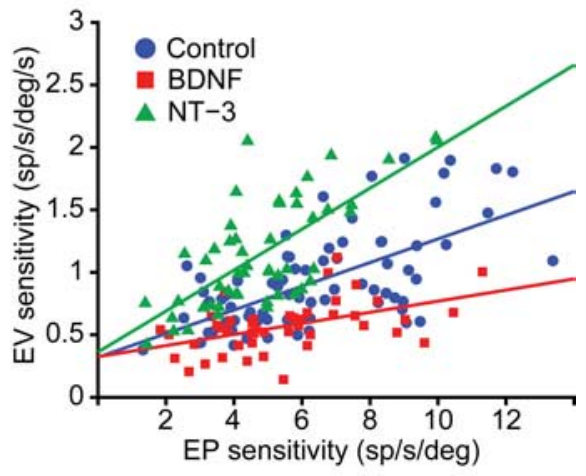

E. NT-3

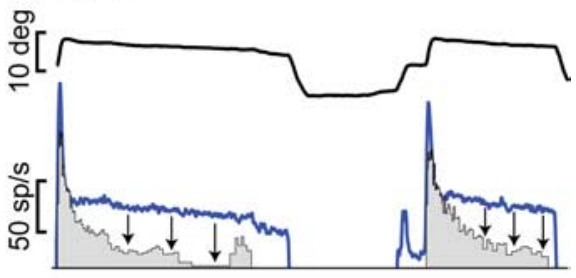

F. BDNF

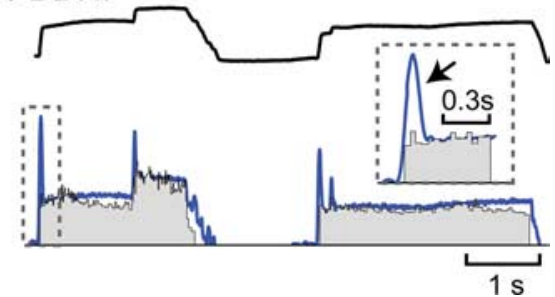

as they exhibited lower $k$ sensitivities for a given $r$ value $(r=0.045 k+0.32 ; r=0.48$; $p<0.001)$. This indicated that despite a robust relationship existing between $k$ and $r$ at the population level, the ratio between both parameters can be scaled as a consequence of the relative synaptic efficacy of both tonic and phasic inputs to the motoneurons depending on the trophic support used.

We calculated the expected firing of motoneurons using the actual parameters of eye movement demonstrating marked contrasts with the actual firing recorded. Thus, treatment with NT-3 revealed that the expected firing of the motoneuron when using the actual $r$ value (Fig. $2 E$, blue lines), and the expected $k$ value from the control group (Fig. 2D, blue), demonstrated a deficit of firing rate during eye fixations (marked by down-directed arrows). In a complementary manner, the treatment with BDNF revealed that the expected bursts for a control motoneuron, having the actual $k$ value of the treated cell but an extrapolated $r$ value from the control population, were much larger than the actual firing recorded (Fig. $2 F$, blue line, better seen in the inset). This discrepancy indicated a disbalance in the proportion of $k$ to $r$ values that scaled differently than in the control group.

Figure 2. Complementary firing patterns of BDNF- and NT-3-treated motoneurons. $A$, Control motoneurons burst for ondirected saccades and fire tonically during eye fixations. $\boldsymbol{B}$, Treatment with BDNF for $8 \mathrm{dimmediately} \mathrm{after} \mathrm{axotomy} \mathrm{reduced} \mathrm{bursts}$ in response to saccades. $\boldsymbol{C}$, Same as $\boldsymbol{B}$, but for a 7-d NT-3-treated cell displaying large bursts for saccades but reduced tonic firing for eye fixations. $\boldsymbol{D}$, Relationship between $k$ and $r$ sensitivities obtained by linear regression analysis for 75 control (blue), 45 BDNF-treated (red) and 51 NT-3-treated (green) motoneurons. The control population (blue) regression line was $r=0.095 k+$ $0.32(r=0.63 ; p<0.001)$, whereas the BDNF-treated population (red) showed a trend toward low $r$ values, and thus the regression line was $r=0.045 k+0.32(r=0.48 ; p<0.001)$. To the contrary, the NT-3 population (green) had higher $r$ values, and the regression line was $r=0.164 k+0.36(r=0.71 ; p<0.001)$. $\boldsymbol{E}$, Response of a motoneuron (gray firing rate) NT-3-treated for $14 \mathrm{~d}$ immediately after axotomy. The blue line represents the firing rate simulation obtained with the actual eye movement and the rvalue but determining the $k$ parameter from the control population shown in $\boldsymbol{D}$. Arrows point to the decay in firing rate during fixations. $\boldsymbol{F}$, Response of a BDNF-treated motoneuron (gray) for $24 \mathrm{~d}$ demonstrating a tonic mode of firing. The blue line as in $\boldsymbol{E}$ represents the simulated firing obtained with the actual eye movements and $k$ but determining $r$ from the control population shown in $\boldsymbol{D}$. The arrow points to the reduced bursts during saccades that are characteristic of BDNF-treated cells that, however, exhibit normal tonic firing.

position sensitivity in control motoneurons demonstrated that a relationship existed and is described by the equation $r=0.095 k$ $+0.32(r=0.63 ; p<0.001)$. Thus, the larger the $k$, the larger was the $r$ value (Fig. $2 D$, blue) with $r$ being $\sim 1 / 10$ th of the $k$ value. As a consequence of the treatment with individual neurotrophins (BDNF or NT-3), the population scatterplot was above the control group after NT-3 treatment (Fig. $2 D$, green), because they were dominated by higher $r$ for a given $k(r=0.164 k+0.36 ; r=$ $0.71 ; p<0.001)$. To the contrary, treatment with BDNF shifted the population scatterplot below the control group (Fig. $2 D$, red)

$\leftarrow$

(Figure legend continued.) +64.2 for the control $(r=0.87 ; p<0.001)$, and FR' $=0.34 \mathrm{EP}+$ 3.0 (dots; $r=0.8 ; p<0.001$ ) for the axotomized motoneuron. $\boldsymbol{F}, \boldsymbol{G}$, Time course of changes in $k$ and $r$ sensitivities after axotomy. Asterisks indicate significant differences with the control value (ANOVA test, Holm-Sidak method for post hoc comparisons, $p<0.01$ ).
Joint administration of BDNF and NT-3 produces recovery of motoneuronal sensitivities to eye movement

A series of experiments consisted in the simultaneous administration of both neurotrophins. As expected from their individual actions, the recovery was significant for both the eye position and the eye velocity sensitivities and for both types of protocol, either immediate or delayed administration after axotomy (Fig. $3 A, B$ ). Firing of motoneurons appeared balanced in burst and tonic modulation, as shown in Figure $4 \mathrm{~A}$ for an example of a motoneuron axotomized for $28 \mathrm{~d}$ and with the immediate trophic support. This result indicated that trophic restoration could be maintained for long-term after lesion and changes could also be seen in the electroneurographic recordings (Fig. $4 A$, middle trace). The effects of the trophic support waned after administration ceased and motoneuron firing appeared again axotomy-like as well as the electroneurographic recording (Fig. 4B). However, trophic support could be restored once the neurotrophin administration resumed and cells turned again into an apparently normal firing pattern with bursts and tonic discharges similar to controls (Fig. $4 C$ ). Thus, the joint administration of BDNF and NT-3 restored the normal firing by scaling the appropriate sensitivities to eye position and velocity of axotomized abducens motoneurons. Removal of the trophic support by stopping the neurotrophin administration led neuronal sensitivities to return to the axotomy levels. 

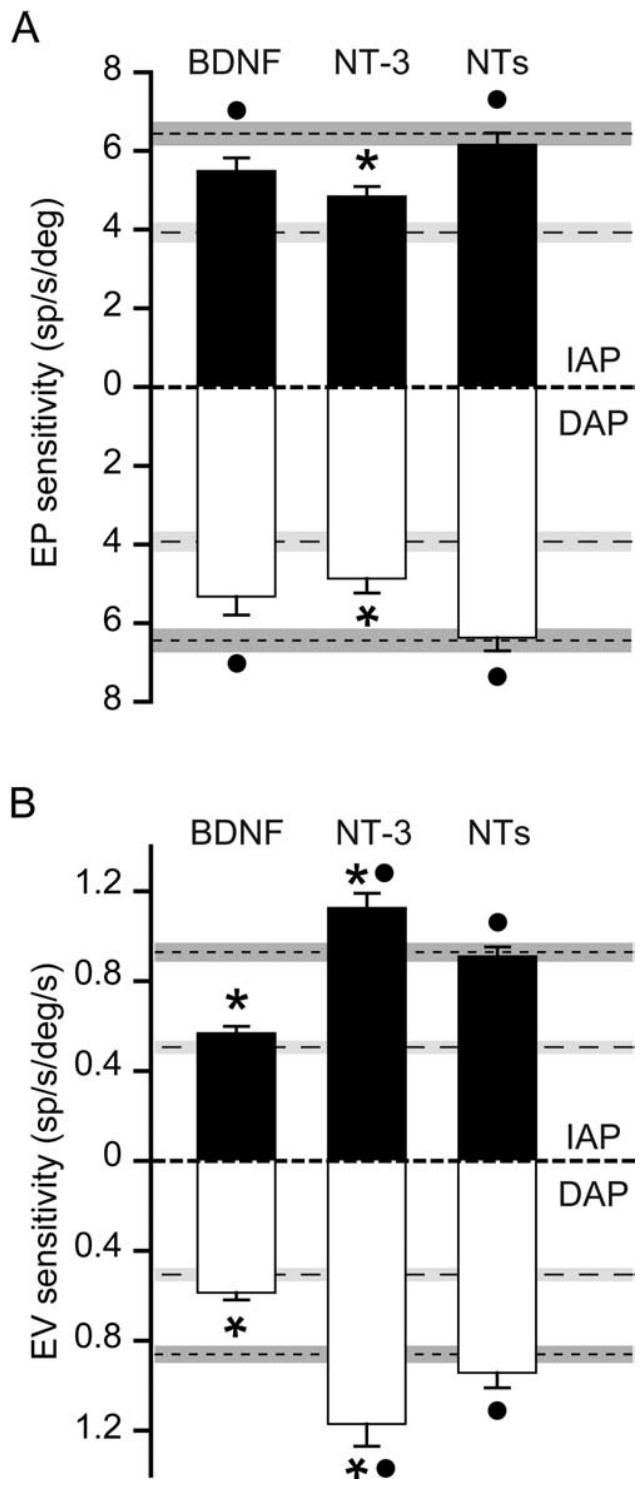

Figure 3. Quantitative effects in neuronal sensitivities induced by the neurotrophin treatments and blockade with K252a. $A$, Eye position sensitivity expressed as mean \pm SEM for 75 control motoneurons (dashed line and gray band represent mean \pm SEM) and 65 axotomized motoneurons (stippled and light gray band). Up-directed bars and error bars represent the mean $k$ values and SEM obtained for 45 (BDNF), 51 (NT-3) and 81 (BDNF+NT-3 labeled as NTs) motoneurons obtained after the IAP. The down-directed bars and error bars represent the mean $k$ values and SEM obtained from 24 (BDNF), 31 (NT-3) and 44 (BDNF+NT-3) motoneurons obtained after the DAP. Asterisks represent statistically significant differences from control group. Note the recovery of $k$ with BDNF or with the mixture of BDNF + NT-3 but not with NT-3. Dots represent statistically significant differences from axotomy group (ANOVA test, HolmSidak method for post hoc comparisons, $p<0.01$ for individual comparisons) $\boldsymbol{B}$, Same as $\boldsymbol{A}$, but for the eye velocity sensitivity. Equal number of cells and significance levels apply as in $\boldsymbol{A}$. Note recovery of eye velocity sensitivity $(r)$ with NT-3 and BDNF + NT-3 but not the recovery with BDNF.

We tested whether the actions of neurotrophins on abducens motoneurons were mediated via the signaling of Trk receptors by using the selective inhibitor of tyrosine kinase action K252a in the presence of a mixture of BDNF and NT-3. Recorded cells showed firing patterns that resembled axotomy when neurotrophins were applied together with the inhibitor from the distal end of sectioned axons (supplemental Fig. 2, available at www.jneurosci. org as supplemental material). The eye position and eye velocity sensitivities under the action of K252a decayed to axotomized
A. $28 \mathrm{~d}$ after BDNF+NT-3
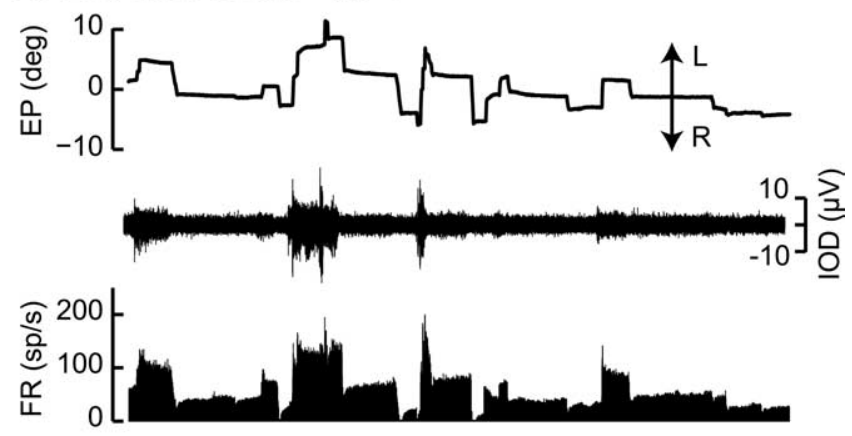

B. 14d after saline
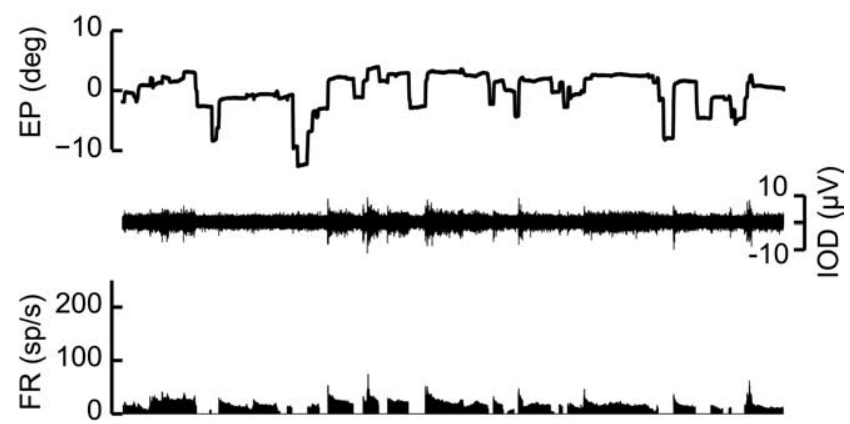

C. $14 \mathrm{~d}$ after treatment resumes
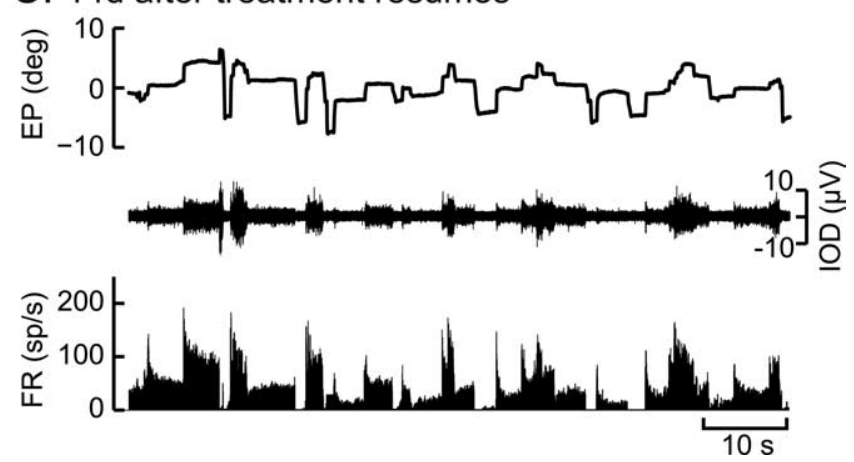

Figure 4. Firing rate restoration induced by treatment with a mixture of BDNF and NT-3. $\boldsymbol{A}$, Motoneuron discharge pattern recorded $28 \mathrm{~d}$ after axotomy and BDNF+NT-3 treatment (immediate administration protocol). In $\boldsymbol{A}-\boldsymbol{C}$, the eye position is shown in the top graph, the middle trace represents the electroneurogram recorded at the stump of the severed abducens nerve indicating that similar changes occurred in the whole nerve, and the bottom represents the instantaneous firing rate. $\boldsymbol{B}$, Another motoneuron recorded in the same animal but $14 \mathrm{~d}$ after suspending the neurotrophic support. Note that this cell was characterized by a low firing rate for both saccades and spontaneous fixations resembling the axotomy state. $C$, Motoneuron recorded in the same animal but $14 \mathrm{~d}$ after resuming treatment showing the restoration of the discharge characteristics toward normal.

values. Motoneurons returned to either control values once the inhibitor was eliminated from the treatment or stayed within the axotomized range of values when administration of neurotrophins stopped.

Neurotrophin-mediated recovery of vestibular synaptic potentials

We tested synaptic potentials of vestibular origin in abducens motoneurons (Baker and Highstein, 1975; Delgado-García et al., 1988; de la Cruz et al., 1994; Pastor et al., 1997). Extracellular synaptic potentials elicited from the ipsilateral vestibular nerve (inhibitory) were a positive wave indicative of hyperpolarizing 
action at the intracellular level. To the contrary, extracellular potentials elicited contralaterally (excitatory) were seen as a negative waves. As a result of axotomy, the vestibular potentials decreased in some cases almost completely although the presynaptic arrival of the afferent volleys were seen (Fig. $5 B$, dots). Treatment with NT-3 produced a significant recovery of the ipsilateral-inhibitory potentials (Fig. 5C,E), whereas treatment with BDNF produced mostly a recovery of contralateralexcitatory-synaptic potentials (Fig. $5 D, E$ ).

We used these stimuli while recording abducens motoneurons in the awake animal to elicit changes in firing probability immediately after application of the stimulus, thus testing synaptic efficacy on treated motoneurons (Broussard et al., 1995). Electrical stimulation at $1.1 \times$ the threshold for detecting eye movements directed toward the contralateral side resulted in transient changes of the discharge rate of abducens motoneurons, that once averaged, produced a characteristic pattern of facilitation and disfacilitation of the discharge rate produced by contralateral and ipsilateral stimuli, respectively (Fig. 6A). For the majority of cells, 200-300 stimuli were used to generate the PSTH. Figure 6, $B-E$, shows the averaged response for each experimental condition using 13-17 cells in each group. Characterization of the bins that were significant using the $z$-score statistics demonstrated that for the bins within the initial 4-5 ms of the response there was a significant facilitation (for contralateral stimulation) or disfacilitation of the response (for ipsilateral stimulation). We quantified the responses through the computation of the area above or below the mean response previous to the stimulation (Fig. 6F, gray areas). Quantification of the results demonstrated that BDNF produced a significant recovery of the excitatory response (Fig. 6G). Contrary to the results found with the extracellular field potentials, NT-3 did not produce a significant recovery of the ipsilateral-inhibitory response, because the tonic resting level was low and the minimum response in a bin is zero, so the carving by inhibition of the PSTH response was not very conspicuous.

The effects of neurotrophin administration to motoneurons studied during the vestibulo-ocular reflex demonstrated that (1) axotomy produced a decay of the neuronal sensitivities to eye position and velocity (supplemental Fig. $3 B, C-F$, available at www.jneurosci.org as supplemental material), (2) NT-3 produced the recovery of eye velocity sensitivity (supplemental Fig. $3 E, F$, available at www.jneurosci.org as supplemental material), (3) BDNF produced the complementary recovery of the eye position sensitivity (supplemental Fig. $3 E, F$, available at www. jneurosci.org as supplemental material), (4) the dual administration produced a full recovery of eye position and velocity sensitivities (supplemental Fig. $3 A, C-F$, available at www.jneurosci. org as supplemental material) that was (5) abolished by the inhibitor K252a (supplemental Fig. 3E,F, available at www. jneurosci.org as supplemental material).

\section{BDNF and NT-3 prevent synaptic stripping}

We found that the functional alterations of axotomy described here were directly related to the degree of synaptic stripping. To quantify these deficits of synaptic coverage, we performed immunohistochemistry for synaptophysin and GFAP which are markers for synaptic boutons and astrocytes, respectively. We also determined the fraction of inhibitory boutons by using immunostaining against VGAT. In the control abducens nucleus, the motoneurons were surrounded in a $48.1 \pm 1.8 \%$ of the somatic perimeter by synaptophysin-IR boutons and $20.1 \pm 1.1 \%$ of the perimeter was covered by astroglial filaments (Fig. 7, control; Fig.
A. Control
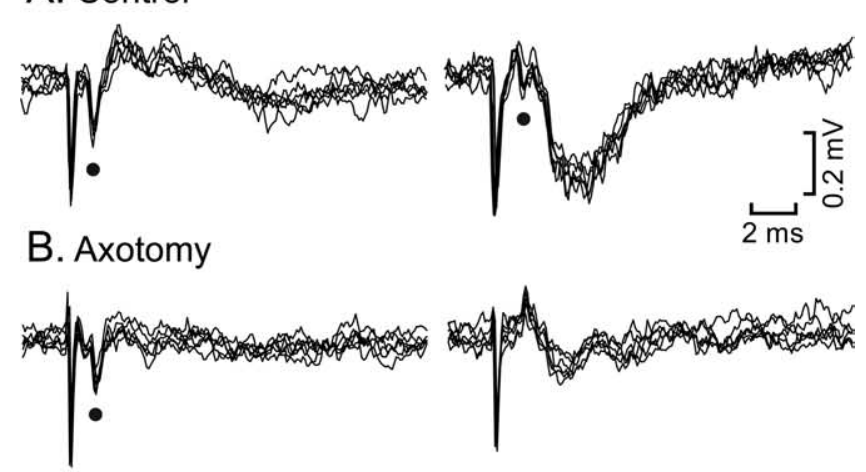

C. NT-3

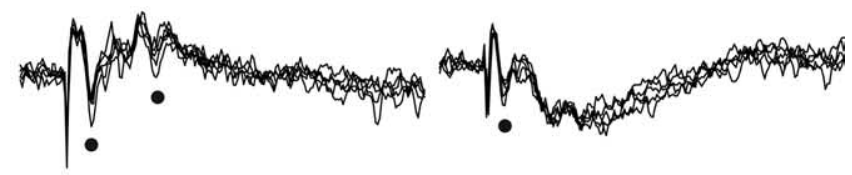

D. BDNF

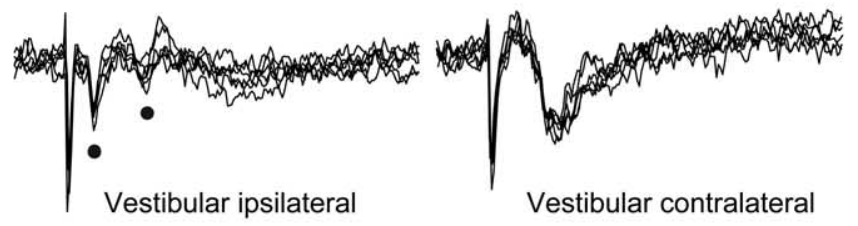

E

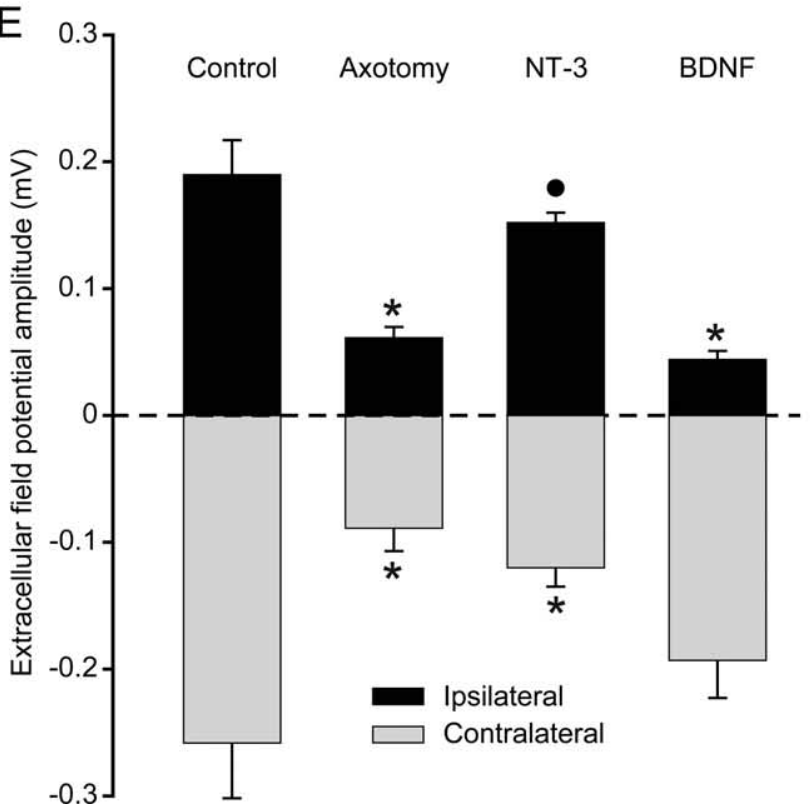

Figure 5. Changes in synaptic potentials. $A-D$, Extracellular field potentials recorded at the center of the abducens nucleus upon single pulse electrical stimulation of the ipsilateral (left side records) or the contralateral (right side records) vestibular nerve. In control $(\boldsymbol{A})$, the inhibitory input produces a positive extracellular wave, whereas the excitatory input produces a negative wave. During axotomy $(\boldsymbol{B})$, both waves are extremely reduced, but note, however, the presynaptic wavelets (dots) indicative of the afferent volley of presynaptic fibers reaching the abducens nucleus. Treatment with NT-3 $(\boldsymbol{C})$ restored the positive wave (inhibitory ipsilateral input), whereas BDNF treatment $(\boldsymbol{D})$ produced the selective recovery of the negative wave (excitatory contralateral input). $\boldsymbol{E}$, Summary of changes in mean amplitude of the field potentials for the different conditions. Mean and SEM are shown for 6-13 measurements taken between $6-12 \mathrm{~d}$ after treatment. Asterisks indicate significant differences with respect to control group (ANOVA test, Holm-Sidak method for post hoc multiple comparisons; $p<0.01$ ). 
8). Approximately $66 \%$ of the somatic boutons were VGAT-IR. The consequence of axotomy on surface coverage was that synaptophysin-IR bouton coverage dropped to $21.4 \pm 2.1 \%$ being a large proportion of boutons VGAT-positive, whereas astrocytic filaments proliferate around the somata and doubled coverage to $40.1 \pm 1.6 \%$ (Fig. 7 , axotomy; Fig. $8 A$ ). Treatment with the mixture of BDNF and NT-3 in the immediate administration protocol prevented the drop of synaptophysin-IR and VGAT-IR boutons (Fig. 8A). An intermediate situation occurred by using a single neurotrophin, either BDNF (Fig. 7, BDNF; Fig. $8 A$ ) or NT-3 (Fig. 7, NT-3; Fig. $8 A$ ). In these cases the prevention of synaptic stripping produced intermediate results since coverage values were both different from control (thus, certain synaptic stripping still occurred) but also different from the axotomy values (Fig. $8 \mathrm{~A}$ ). Neurotrophin treatment with either single neurotrophins or the mixture of two neurotrophins diminished in all cases the astrocytic reaction around the somata of motoneurons (Fig. $8 B$ ).

Mean gray value, as a measure of optical density, measured in the neuropil produced a similar pattern of synaptic coverage with synaptophysin-IR and VGAT-IR profiles. The somatic coverage of motoneurons with GFAP was between the control and the axotomy values for the NT-3 treatment and showed a full recovery for the BDNF or the mixture of neurotrophins in the immediate administration protocol (Fig. $8 \mathrm{~B}$ ). The GFAP-IR profiles, however, demonstrated that reactive astrocytes were present in the neuropil for all treatments; therefore, the recovery was partial for treatment with a single neurotrophin but recovery was more profound for treatment with the mix of both neurotrophins (Fig. $8 \mathrm{~B}$ ). Thus, astrocytes were reactive as a consequence of the axotomy in the neuropil but locally withdrew from the surface of the motoneurons concomitantly with the lack of synaptic stripping.

\section{Neurotrophin treatment promotes} afferent bouton reinnervation

The delayed administration protocol of the mixture of neurotrophins allowed us to test the hypothesis that neurotrophins would have a reinnervation effect once the synaptic stripping had taken place. For doing so, abducens motoneurons were axotomized and $15 \mathrm{~d}$ later, once the stripping has taken place, treatment with neurotrophins started until the animals were killed after $30 \mathrm{~d}$. The delayed administration protocol produced the largest recovery of bouton coverage (51.8 \pm $1.57 \%$ ) around motoneuron somata with synaptophysin-IR pro-

$\mathrm{F}$
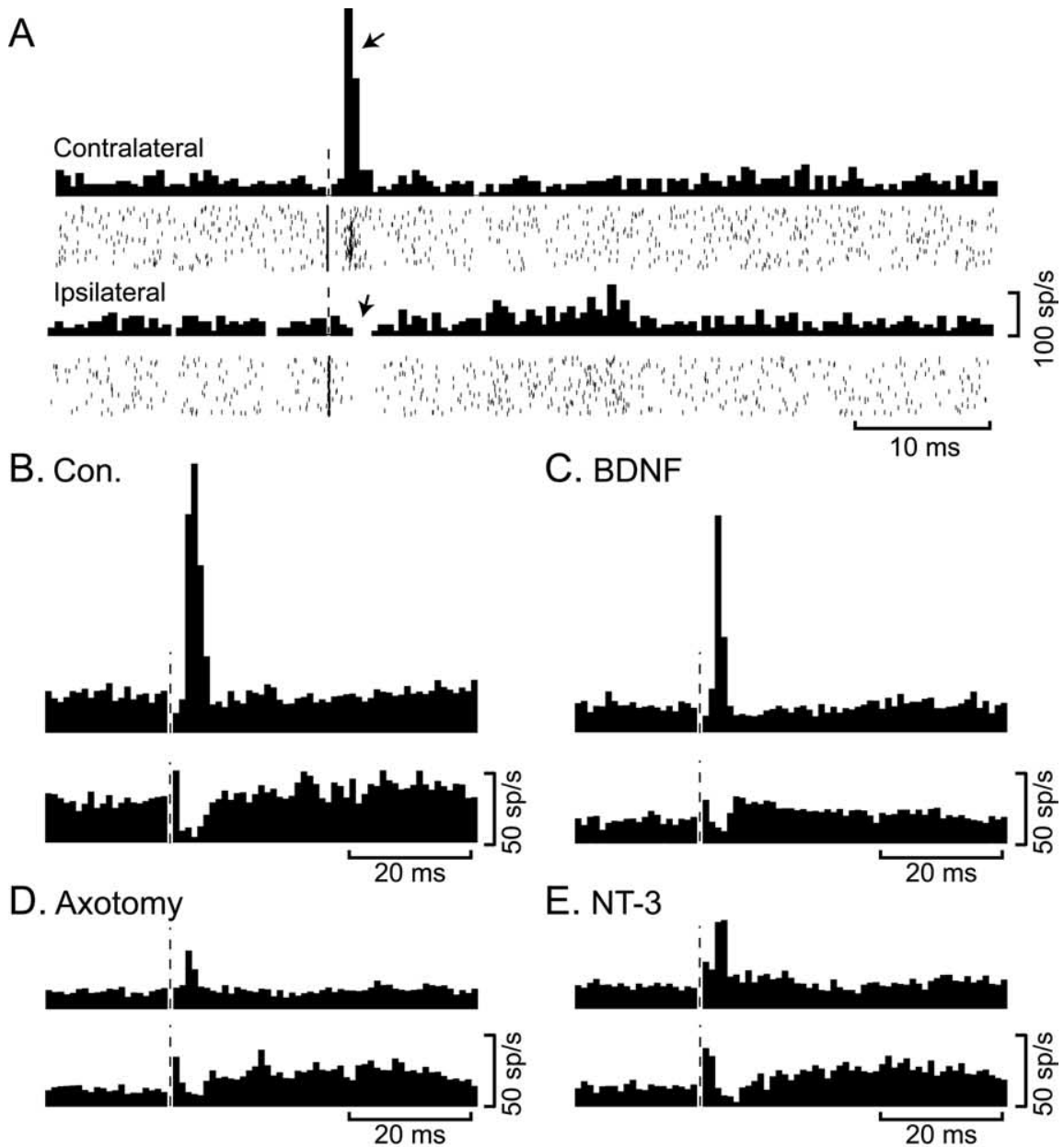

C. BDNF
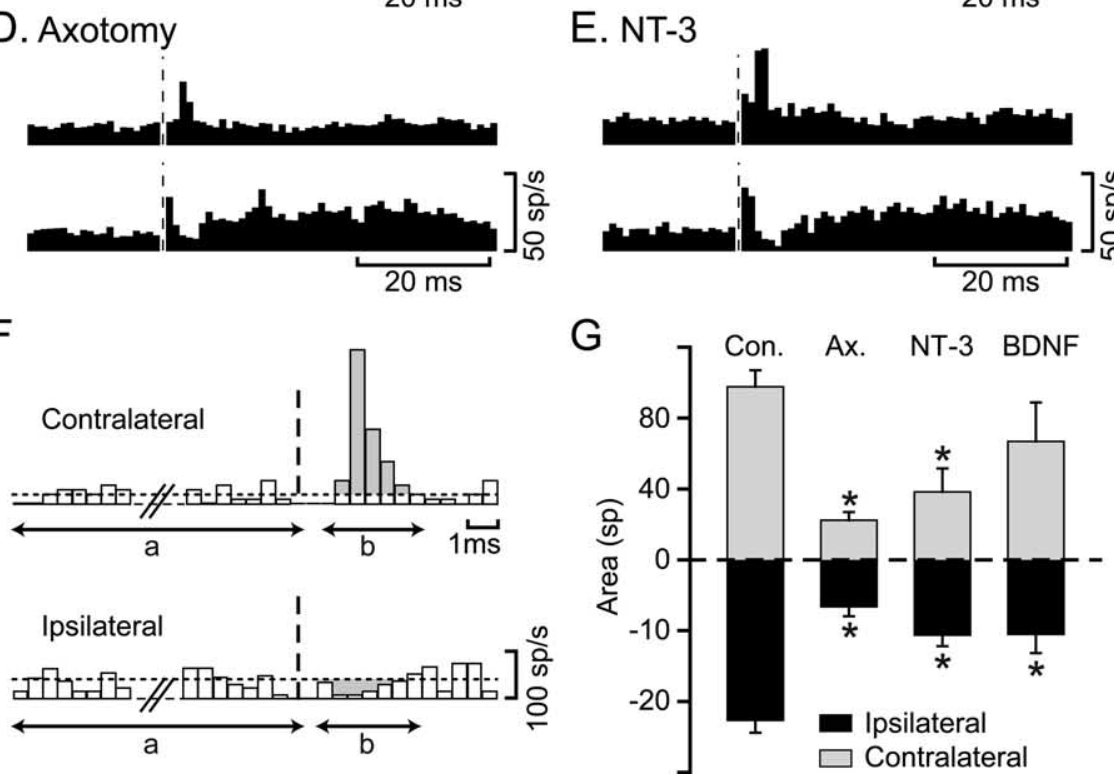

Figure 6. Alterations by axotomy and neurotrophin treatment in abducens motoneuron responses to vestibular nerve electrical stimulation. A, PSTHs constructed with 311 (contralateral) and 283 sweeps (ipsilateral), shown below in raster mode, that display $20 \mathrm{~ms}$ before the stimulus (stimulus artifact bin has been deleted from $\boldsymbol{A}-\boldsymbol{F}$ represented as a vertical dashed line) and $50 \mathrm{~ms}$ after the stimulus. Top PSTH indicates a firing facilitation peaking at $2.5 \mathrm{~ms}$ (arrow) upon contralateral stimulation, and the lower PSTH shows a firing disfacilitation that is maximal at $2 \mathrm{~ms}$ (arrow) after the ipsilateral stimulation. Bin width for PSTH is $0.5 \mathrm{~ms}$. $\boldsymbol{B}-\boldsymbol{E}$, PSTH for contralateral (top) and ipsilateral (bottom) vestibular nerve stimulation averaged for 13-17 responses of individual cells normalized to the number of sweeps. $\boldsymbol{F}$, Area measurement (in spikes) consisted in obtaining the number of counts (in spikes/s) times the bin width (in seconds) of the initial eight bins excluded the first one $(\boldsymbol{b})$ that were either higher, for contralateral stimulation, or lower, for ipsilateral vestibular stimulation than the mean firing of the 20 ms previous to the stimulus application (a). G, Quantification of the PSTH responses demonstrated a significant reduction in the area of the response for axotomized motoneurons and a significant recovery of the PSTH amplitude for contralateral vestibular stimulation after BDNF treatment. Asterisks indicate significant differences with control groups (ANOVA test; Holm-Sidak method for multiple comparisons; $p<0.01$ ).

files (Fig. 7, BDNF+NT-3; Fig. 8A). Compatible with these results, the coverage with GFAP-IR profiles was the lowest of all treatments $(15.9 \pm 0.9 \%)$. In the neuropil, the mix of BDNF and NT-3 in the delayed administration protocol produced also the 


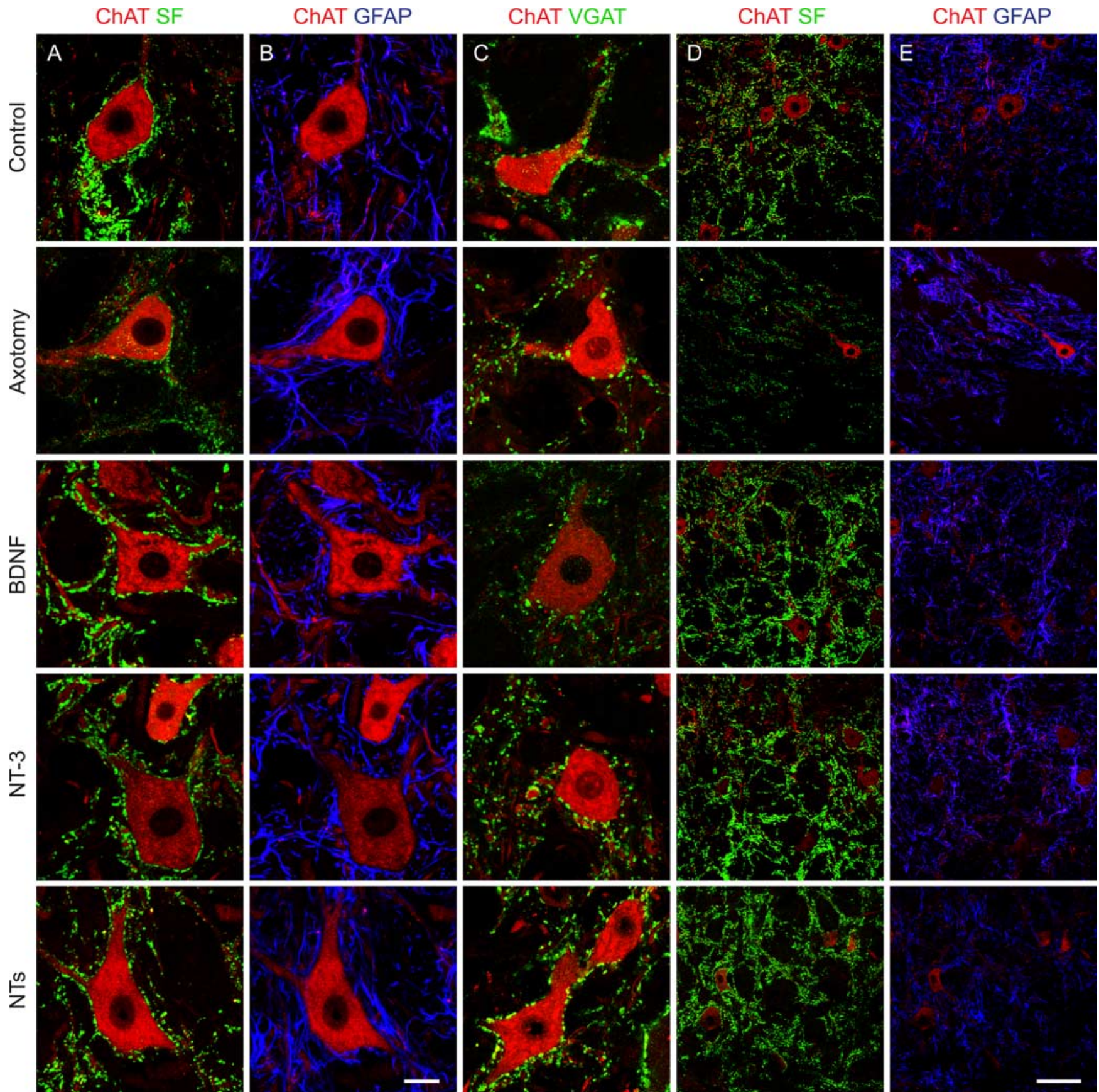

Figure 7. Neurotrophin involvement in prevention of synaptic stripping. $A$, Confocal high-magnification images of motoneurons in control, axotomy, treated with BDNF, NT-3 and treated with BDNF+NT-3 (NTs) illustrating the synaptophysin innervation in green and the motoneuronal cell body in red (ChAT). All sections were obtained from animals after $15 \mathrm{~d}$ of axotomy alone or after treatment with neurotrophins in an immediate administration protocol. Note the marked reduction of boutons after $15 \mathrm{~d}$ of axotomy. $\boldsymbol{B}$, Same motoneurons and treatments as in $\boldsymbol{A}$ but for GFAP coverage. Note an intense proliferation of astrocytic filaments surrounding the motoneuron somata. C, Confocal images of VGAT labeling in ChAT identified motoneurons. $\boldsymbol{D}, \boldsymbol{E}$, Same as $\boldsymbol{A}, \boldsymbol{B}$, but for confocal low-magnification images illustrating the effects of treatments on boutons and glial filaments in the abducens nucleus neuropil. Scale bars: $(\boldsymbol{A}-\boldsymbol{C}) 10 \mu \mathrm{m} ;(\boldsymbol{D}, \boldsymbol{E}) 40 \mu \mathrm{m}$.

largest recovery in the optical density (Fig. $8 \mathrm{~B}$ ). These results indicate that neurotrophins used here have a profound synaptotrophic and synaptotropic effect on axotomized motoneurons.

\section{Discussion}

Present data show that BDNF and NT-3 administered to the severed axons of motoneurons results in both the prevention of stripping of synaptic afferents when immediately administered after lesion and the recovery of afferent innervation after synaptic stripping due to lesion. Axotomy also induced the decay in neuronal sensitivities to eye movements and a reduction in the amplitude of synaptic potentials of vestibular origin that were also restored to normal values when both neurotrophins were applied. Moreover, the discharge characteristics recovered in a complementary manner when only one neurotrophin was administered. Thus, it is shown the selective trophic dependence of bursting firing on NT-3 whereas BDNF supported the tonic firing of motoneurons. The present data support a link between the synaptotrophic actions of neurotrophins retrogradely delivered and the alter- 


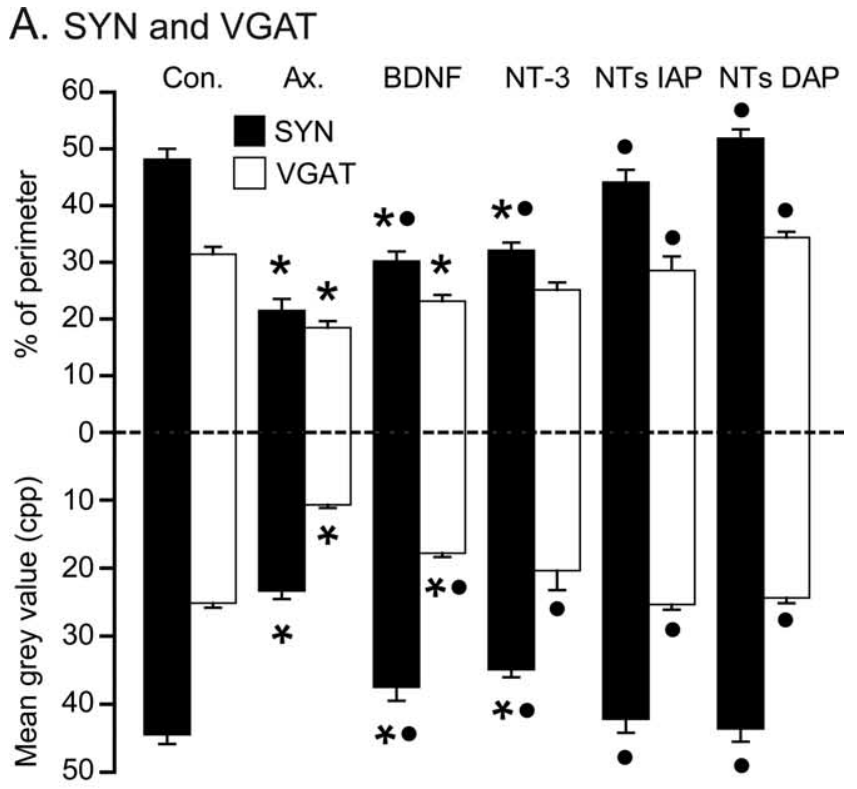

B. GFAP

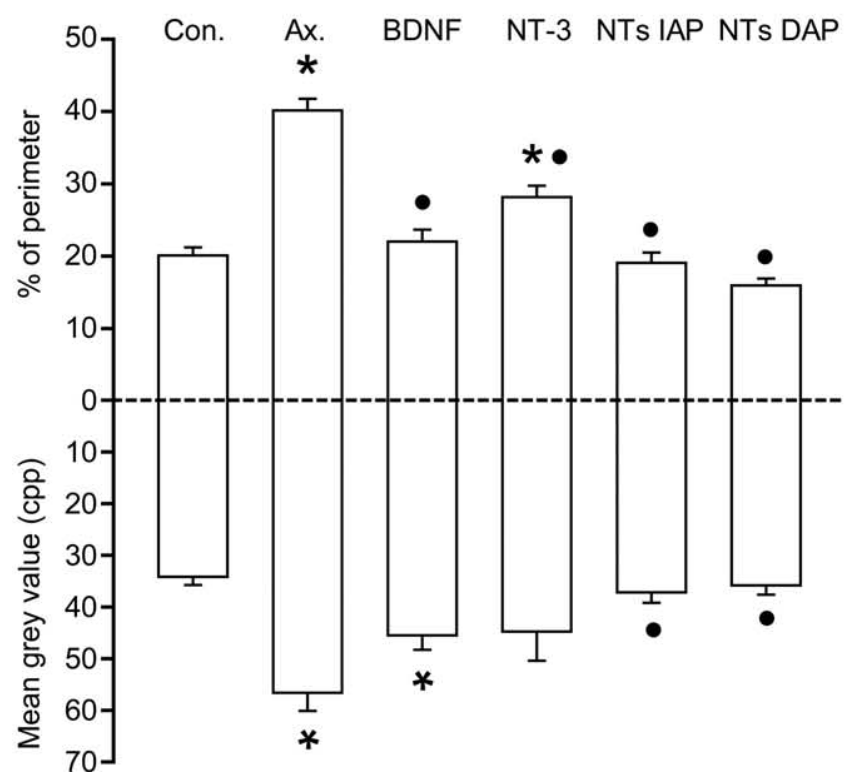

Figure 8. Prevention of synaptic stripping and promotion of reinnervation by neurotrophins. $\boldsymbol{A}$, Changes in synaptophysin-IR (green) and VGAR-IR (red) boutons in the abducens nucleus after axotomy and different treatments. Up-directed bars represent the percentage of covered perimeter of the motoneurons in control, axotomy, single neurotrophin treatment (BDNF or NT-3) and double neurotrophin treatment for either IAP or DAP. Down-directed bars correspond to measurements in the neuropil of the same variables. Bars represent mean $\pm \mathrm{SEM}$ for 15-46 motoneuronal profiles in each group and 26-50 measurements in the neuropil. $\boldsymbol{B}$, Same as $\boldsymbol{A}$, but for measurements of GFAP-IR profiles around somata (up-directed bars) or in the neuropil (down-directed bars). Asterisks indicate significant differences with control, whereas dots indicate significant differences with axotomy. Two-way ANOVA, Holm-Sidak method for pairwise multiple comparisons ( $p<0.01$ ).

ations of neuronal firing patterns during oculomotor behaviors through plastic rearrangements of synaptic contacts.

\section{Alterations of target-derived trophic support determine the morphophysiological changes induced by axotomy}

Nerve injury induces profound changes at the structural and functional level of damaged motoneurons (Sumner, 1975; Mendell, 1984). It is well known that these changes in electrical and synaptic properties revert with target reinnervation (DelgadoGarcía et al., 1988; Brännström and Kellerth, 1999) and remain altered when reinnervation is prevented (Sumner, 1976; Pinter and Vanden Noven, 1989; Vanden Noven and Pinter, 1989). Thus, for preventing random target reinnervation and/or reestablishment of trophic support from orbital tissues, we devised a method for controlling the extracellular environment that bathes the axonal stumps (Davis-López de Carrizosa et al., 2008). We cannot ascertain whether neurotrophins signaled to afferents through transsynaptic translocation, like tetanus toxin does (González-Forero et al., 2005; Rind et al., 2005), or induced in the motoneurons the signal for alterations in their synaptic coverage.

The cascade of events mediated by neurotrophins was initiated at Trk receptors located at the axonal stump and abolished by K252a, a selective inhibitor in vivo and in vitro of Trk action (Rutherford et al., 1997; Rattiner et al., 2004; Bozdagi et al., 2008). In our system, K252a led to decreased neuronal sensitivities to eye movement parameters that resembled those found during axotomy, and thus it can be concluded that this agent blocked the exogenous trophic support. It can be stressed the idea that exogenous trophic support switches the cellular program that maintains the axotomized cell synaptically disconnected from the afferent circuit to a cellular program which implies the reinnervation by afferents. Previous findings in the axotomized abducens motoneurons and internuclear neurons, also in the cat, support the idea of a strong dependence of these neurons on their target as demonstrated by the recovery of firing properties in coincidence with target reinnervation (Baker et al., 1981; Delgado-García, 1988; Benítez-Temiño et al., 2005).

\section{Neurotrophin complementary action on specific inputs to motoneurons}

Our findings show that neurotrophic support starting from the day of lesion prevents the withdrawal of synaptic inputs and that protection against synaptic stripping is more effective when BDNF and NT-3 were applied together rather than when only one neurotrophin alone was used. These results could indicate either an additive effect over all the different afferents to the motoneuron or a complementary effect over distinct afferents. Our neuronal recordings demonstrate the second alternative. Thus, NT-3 supported the phasic firing of motoneurons whereas BDNF supported the tonic firing. BDNF and NT-3 have a strong synaptotrophic action regulating the synaptic composition and the growth of presynaptic and postsynaptic elements in the adult brain (Novikov et al., 2000; Cotrufo et al., 2003) and in vitro (McAllister et al., 1997; Je et al., 2006). It is noteworthy that BDNF and NT-3 frequently have opposing roles that account for the specification of electrophysiological properties in the spiral ganglion neurons (Adamson et al., 2002; Zhou et al., 2005) to the growth of dendrites in layer 4 pyramidal neurons (McAllister et al., 1997).

NT-3 produced the recovery of the phasic firing during spontaneous saccades and increased the synaptic coverage of axotomized abducens motoneurons. The phasic component is provided by burst reticular neurons (excitatory and inhibitory; EBN, IBN) located in the paramedian pontine reticular formation (Highstein et al., 1976; Hikosaka et al., 1978; Igusa et al., 1980) that convey a strong burst during on-directed saccades and a pause during movements in the opposite direction (Büttner and Büttner-Ennever, 2006). It is likely that NT-3 exerts a trophic effect over reticular synaptic contacts on motoneurons that explains the robust bursts during saccades (Fig. 9). Similarly the presence of strong pauses associated to off-directed saccades in- 
IPSILATERAL

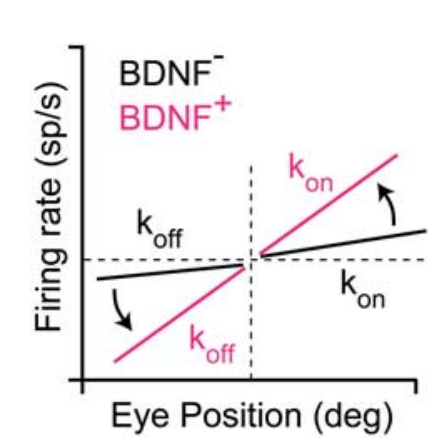

\section{CONTRALATERAL}

BDNF

NT-3
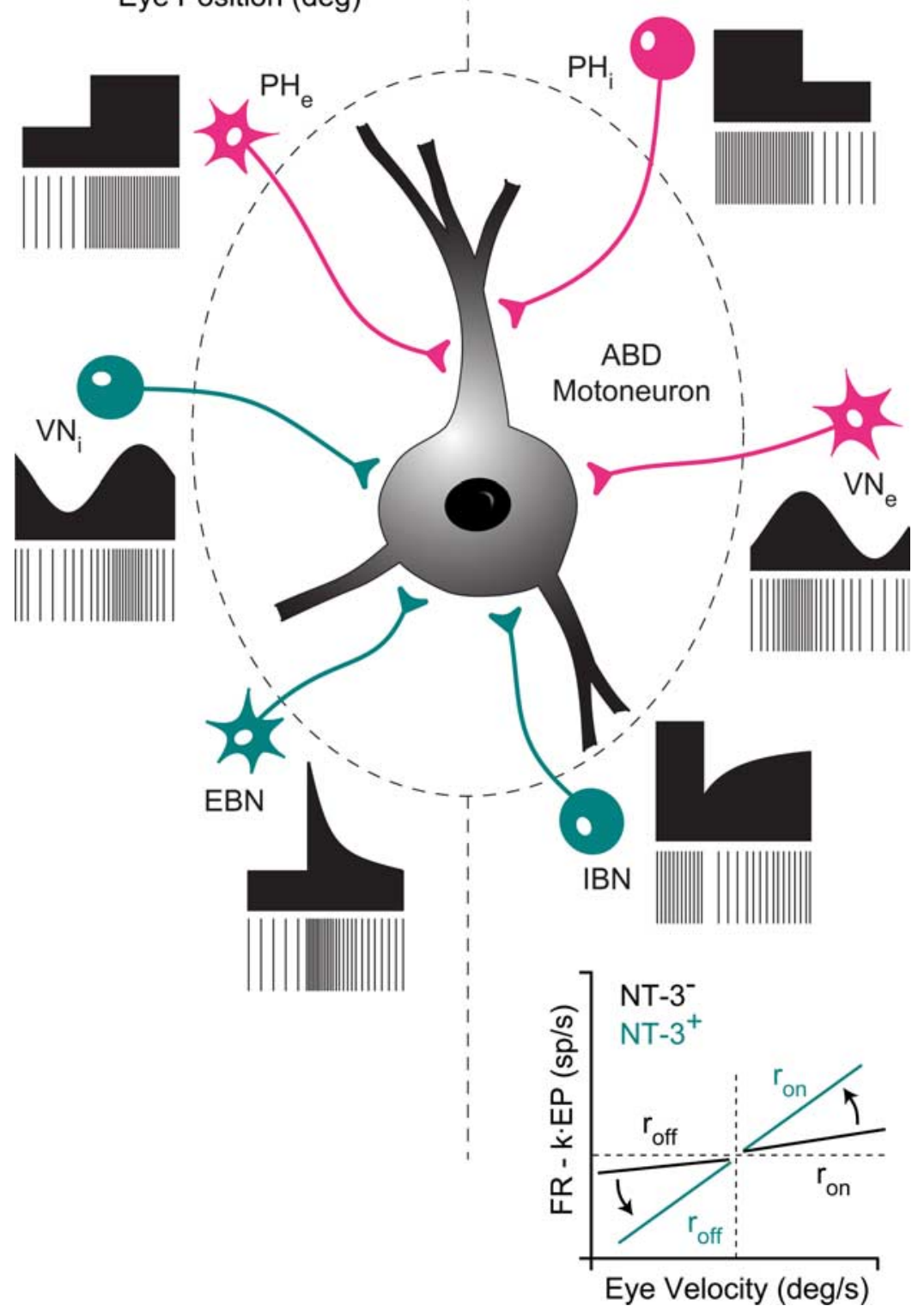

Figure 9. Diagram indicating trophic actions of BDNF and NT-3 on abducens motoneurons afferents. Abducens motoneurons receive a main set of three bilateral and reciprocal afferents that convey signals for conjugate eye movements. Inputs represented to the right of the figure are ipsilateral. Trophic actions of BDNF and NT-3 are labeled in pink and green, respectively. See discussion for further details.

dicates that also the inhibitory burst neurons are trophically maintained by NT-3, otherwise the rate-velocity plots found would have a smaller slope (Fig. 9). Interestingly, eye velocity sensitivity resulted after NT-3 treatment higher than in control motoneurons. A similar result occurs in the Ia synapses over axotomized spinal motoneurons in vitro and in vivo. (Munson et al., 1997; Arvanian et al., 2003). NT-3 derived from muscle spindles or after NT-3 treatment increases the amplitude of this synapse over the control value, but results impaired for sustained high frequency firing (Mendell et al., 1999; Chen et al., 2002).

BDNF induced the recovery of the tonic firing of axotomized abducens motoneurons as well as an increase in their synaptic coverage. The tonic component arises from the output of a velocity-toposition integrator (Aksay et al., 2001) whose main output cells are the bursttonic neurons of the prepositus hypoglossi nucleus (Escudero et al., 1992) that provide a reciprocal tonic excitatory/inhibitory drive during on- and off-directed eye fixations, respectively (Fig. 9). Chronic treatment with BDNF has been shown to produce a long-term enhancement of synaptic transmission with no changes in voltage-gated sodium and potassium currents (Sherwood and Lo, 1999). Nonetheless, it has been shown that BDNF increases the excitability to depolarizing and hyperpolarizing currents through modulation of potassium currents rendering tonic and rebounding cells in response to current steps (Youssoufian and Walmsey, 2007). BDNF, also in vitro, decreases membrane potential fluctuations promoting rhythmical firing through a K252asensitive mechanism that increases a persistent sodium current (Fujisawa et al., 2004) that others have identified as a tetrodotoxin-insensitive sodium current (Blum et al., 2002). BDNF has also been shown to regulate the synaptic composition of axotomized motoneurons (Novikov et al., 2000). From the signal analysis of firing rate we found that both the $k$-on and the $k$-off sensitivities were both restored to control values after BDNF treatment alone or in combination with NT-3. This result indicates a trophic action of BDNF over the tonic excitatory and inhibitory input to the motoneurons. If that were not the case, the rate-position plots used to obtain the eye position sensitivity would have a smaller slope than that found (Fig. 9).

A third major source of afferents to the abducens nucleus arises from the medial vestibular nucleus that provides reciprocal, excitatory-contralateral/inhibitoryipsilateral input to the abducens motoneurons (Fig. 9). The firing of abducens neurons during the VOR can be expressed as the sum of a vestibular input and the output of a velocity-to-position integrator that provides abducens neurons a signal related to eye position. The vestibular signal is channeled through the position-vestibular-pause neurons (Ishizuka et al., 
1980, McCrea et al., 1980) and the tonic signal is also mainly channelled through prepositus hypoglossi and these vestibular neurons (Ramachandran and Lisberger, 2006). We found excitatory vestibular synaptic potentials recovered with BDNF as well as the amplitude of modulation in the PSTH after contralateral vestibular stimulation, indicating that the input from excitatory vestibular neurons contralateral to the abducens would be dependent on the trophic action of BDNF. However, inhibitory vestibular synaptic potentials recovered with NT-3 treatment indicating that the ipsilateral vestibular neurons would be dependent on trophic support from NT-3 (Fig. 9).

The present work opens the possibility for the existence of different motoneuronal types (tonic, phasic) according to their responsiveness to different mixtures of neurotrophins coming from the target. Our previous study (Benítez-Temiño et al., 2004) demonstrated the coexpression of TrkB and TrkC receptors in $72-82 \%$ of abducens motoneurons which also implies that $18-$ $28 \%$ of the motoneurons might depend solely on either BDNF or NT-3. This would indicate that some control motoneurons might be purely tonic, others purely phasic and the vast majority tonicphasic. This likely heterogeneity among motoneurons is in contrast, although, with the homogeneous firing patterns found in abducens motoneurons since every cell demonstrates a tonic and a phasic firing (Delgado-Garcia et al., 1986) (present data). Nonetheless, at least two types of motoneurons have been proposed recently on the basis of retrograde and transneuronal tracing (i.e., twitch large cells and small, nontwitch, peripherally located in the nucleus) (Büttner-Ennever et al. 2001; Ugolini et al., 2006). Despite the fact that smaller, nontwitch motoneurons, having thus higher input resistance, could have a similar sensitivity to saccades arising from a reduced number of reticular boutons, our data suggest that twitch and nontwitch motoneurons might express a different number of TrkB and TrkC receptors and/or receive different amounts of BDNF and NT-3 neurotrophins from their innervated muscle fibers, which in turn would modulate the synaptic coverage and firing patterns of abducens motoneurons.

\section{References}

Adamson CL, Reid MA, Davis RL (2002) Opposite action of brain-derived neurotrophic factor and neurotrophin-3 on firing features and ion channel composition of murine spiral ganglion neurons. J Neurosci 22:1385-1396.

Aksay E, Gamkrelidze G, Seung HS, Baker R, Tank DW (2001) In vivo intracellular recording and perturbation of persistent activity in a neural integrator. Nat Neurosci 4:184-193.

Arvanian VL, Horner PJ, Gage FH, Mendell LM (2003) Chronic neurotrophin-3 strengthens synaptic connections to motoneurons in the neonatal rat. J Neurosci 23:8706-8712.

Baker R, Highstein SM (1975) Physiological identification of interneurons and motoneurons in the abducens nucleus. Brain Res 91:292-298.

Baker R, Delgado-García JM, McCrea R (1981) Morphological and physiological effects of axotomy on cat abducens motoneurons. In: Lesioninduced neuronal plasticity in sensorimotor systems (Flohr H, Precht W, eds), pp 51-63. Berlin: Springer.

Benítez-Temiño B, Morcuende S, Mentis GZ, de la Cruz RR, Pastor AM (2004) Expression of Trk receptors in the oculomotor system of the adult cat. J Comp Neurol 473:538-552.

Benítez-Temiño B, de la Cruz RR, Tena JJ, Pastor AM (2005) Cerebellar grafting in the oculomotor system as a model to study target influence on adult neurons. Brain Res Brain Res Rev 49:317-329.

Blum R, Kafitz KW, Konnerth A (2002) Neurotrophin-evoked depolarization requires the sodium channel $\mathrm{Na}_{\mathrm{v}}$ 1.9. Nature 419:687-693.

Bozdagi O, Rich E, Tronel S, Sadahiro M, Patterson K, Shapiro ML, Alberini CM, Huntley GW, Salton SR (2008) The neurotrophin-inducible gene Vgf regulates hippocampal function and behavior through a brain- derived neurotrophic factor-dependent mechanism. J Neurosci 28:9857-9869.

Brännström T, Kellerth JO (1999) Recovery of synapses in axotomized adult cat spinal motoneurons after reinnervation into muscle. Exp Brain Res 125:19-27.

Broussard DM, DeCharms RC, Lisberger SG (1995) Inputs from the ipsilateral vestibular apparatus to behaviorally characterized abducens neurons in rhesus monkeys. J Neurophysiol 74:2445-2459.

Büttner U, Büttner-Ennever JA (2006) Present concepts of oculomotor organization. In: Neuroanatomy of the oculomotor system (BüttnerEnnever JA, ed), pp 1-42. Amsterdam: Elsevier.

Büttner-Ennever JA, Horn AK, Scherberger H, D’Ascanio P (2001) Motoneurons of twitch and nontwitch extraocular muscle fibers in the abducens, trochlear, and oculomotor nuclei of monkeys. J Comp Neurol 438:318-335.

Caleo M, Medini P, von Bartheld CS, Maffei L (2003) Provision of brainderived neurotrophic factor via anterograde transport from the eye preserves the physiological responses of axotomized geniculate neurons. J Neurosci 23:287-296.

Chen HH, Tourtellotte WG, Frank E (2002) Muscle spindle-derived neurotrophin 3 regulates synaptic connectivity between muscle sensory and motor neurons. J Neurosci 22:3512-3519.

Cotrufo T, Viegi A, Berardi N, Bozzi Y, Mascia L, Maffei L (2003) Effects of neurotrophins on synaptic protein expression in the visual cortex of darkreared rats. J Neurosci 23:3566-3571.

Curtis R, Tonra JR, Stark JL, Adryan KM, Park JS, Cliffer KD, Lindsay RM, DiStefano PS (1998) Neuronal injury increases retrograde axonal transport of neurotrophins to spinal sensory neurons and motor neurons via multiple receptor mechanisms. Mol Cell Neurosci 12:105-118.

Davis-López de Carrizosa MA, Tena JJ, Benítez-Temiño B, Morado-Díaz CJ, Pastor AM, de la Cruz RR (2008) A chronically-implantable device for the controlled delivery of substances, the stimulation and the recording of activity in severed nerves. J Neurosci Methods 167:302-309.

de la Cruz RR, Pastor AM, Delgado-García JM (1994) Effects of target depletion on adult mammalian central neurons: functional correlates. Neuroscience 58:81-97.

Delgado-Garcia JM, del Pozo F, Baker R (1986) Behavior of neurons in the abducens nucleus of the alert cat-I. Motoneurons. Neuroscience 17:929-952.

Delgado-García JM, del Pozo F, Spencer RF, Baker R (1988) Behavior of neurons in the abducens nucleus of the alert cat-III. Axotomized motoneurons. Neuroscience 24:143-160.

Dumoulin A, Rostaing P, Bedet C, Lévi S, Isambert MF, Henry JP, Triller A, Gasnier B (1999) Presence of vesicular inhibitory amino acid transporter in GABAergic and glycinergic synaptic terminals. J Cell Sci 112:811-823.

Escudero M, de la Cruz RR, Delgado-García JM (1992) A physiological study of vestibular and prepositus hypoglossi neurones projecting to the abducens nucleus in the alert cat. J Physiol 458:539-560.

Fuchs AF, Robinson DA (1966) A method for measuring horizontal and vertical eye movement chronically in the monkey. J Appl Physiol 21:1068-1070.

Fujisawa S, Yamada MK, Nishiyama N, Matsuki N, Ikegaya Y (2004) BDNF boosts spike fidelity in chaotic neural oscillations. Biophys J 86:1820-1828.

González-Forero D, Pastor AM, Delgado-García JM, de la Cruz RR, Alvarez FJ (2004) Synaptic structural modification following changes in activity induced by tetanus neurotoxin in cat abducens neurons. J Comp Neurol 471:201-218.

González-Forero D, Morcuende S, Alvarez FJ, de la Cruz RR, Pastor AM (2005) Transynaptic effects of tetanus neurotoxin in the oculomotor system. Brain 128:2175-2188.

Highstein SM, Maekawa K, Steinacker A, Cohen B (1976) Synaptic input from the pontine reticular nuclei to abducens motoneurons and internuclear neurons in the cat. Brain Res 112:162-167.

Hikosaka O, Igusa Y, Nakao S, Shimazu H (1978) Direct inhibitory synaptic linkage of pontomedullary reticular burst neurons with abducens motoneurons in the cat. Exp Brain Res 33:337-352.

Igusa Y, Sasaki S, Shimazu H (1980) Excitatory premotor burst neurons in the cat pontine reticular formation related to the quick phase of vestibular nystagmus. Brain Res 182:451-456.

Ishizuka N, Mannen H, Sasaki S, Shimazu H (1980) Axonal branches and 
terminations in the cat abducens nucleus of secondary vestibular neurons in the horizontal canal system. Neurosci Lett 16:143-148.

Je HS, Yang F, Zhou J, Lu B (2006) Neurotrophin 3 induces structural and functional modification of synapses through distinct molecular mechanisms. J cell Biol 175:1029-1042.

McAllister AK, Katz LC, Lo DC (1997) Opposing roles for endogenous BDNF and NT-3 in regulating cortical dendritic growth. Neuron 18:767778.

McAllister AK, Katz LC, Lo DC (1999) Neurotrophins and synaptic plasticity. Annu Rev Neurosci 22:295-318.

McCrea RA, Yoshida K, Berthoz A, Baker R (1980) Eye movement related activity and morphology of second order vestibular neurons terminating in the cat abducens nucleus. Exp Brain Res 40:468-473.

Mendell LM (1984) Modifiability of spinal synapses. Physiol Rev 64:260-324.

Mendell LM, Johnson RD, Munson JB (1999) Neurotrophin modulation of the monosynaptic reflex after peripheral nerve transection. J Neurosci 19:3162-3170.

Munson JB, Johnson RD, Mendell LM (1997) NT-3 increases amplitude of EPSPs produced by axotomized group Ia afferents. J Neurophysiol 77:2209-2212.

Novikov LN, Novikova LN, Holmberg P, Kellerth J-O (2000) Exogenous brain-derived neurotrophic factor regulates the synaptic composition of axonally lesioned and normal adult rat motoneurons. Neuroscience 100:171-181.

Omura T, Sano M, Omura K, Hasegawa T, Doi M, Sawada T, Nagano A (2005) Different expressions of BDNF, NT3, and NT4 in muscle and nerve alter various types of peripheral nerve injuries. J Peripher Nerv Syst 10:293-300.

Pastor AM, Moreno-López B, De La Cruz RR, Delgado-García JM (1997) Effects of botulinum neurotoxin type $\mathrm{A}$ on abducens motoneurons in the cat: ultrastructural and synaptic alterations. Neuroscience 81:457-478.

Pinter MJ, Vanden Noven S (1989) Effects of preventing reinnervation on axotomized spinal motoneurons in the cat. I. Motoneuron electrical properties. J Neurophysiol 62:311-324.

Ramachandran R, Lisberger SG (2006) Transformation of vestibular signals into motor commands in the vestibuloocular reflex pathway of monkeys. J Neurophysiol 96:1061-1074.

Rattiner LM, Davis M, French CT, Ressler KJ (2004) Brain-derived neurotrophic factor and tyrosine kinase receptor B involvement in amygdaladependent fear conditioning. J Neurosci 24:4796-4806.

Rind HB, Butowt R, von Bartheld CS (2005) Synaptic targeting of retrogradely transported trophic factors in motoneurons: comparison of glial cell line-derived neurotrophic factor, brain-derived neurotrophic factor, and cardiotrophin-1 with tetanus toxin. J Neurosci 25:539-549.

Rutherford LC, DeWan A, Lauer HM, Turrigiano GG (1997) Brain-derived neurotrophic factor mediates the activity-dependent regulation of inhibition in neocortical neurons. J Neurosci 17:4527-4535.

Schinder AF, Poo M-M (2000) The neurotrophic hypothesis for synaptic plasticity. Trends Neurosci 23:639-645.

Sherwood NT, Lo DC (1999) Long-term enhancement of central synaptic transmission by chronic brain-derived neurotrophic factor treatment. J Neurosci 19:7025-7036.

Steljes TP, Kinoshita Y, Wheeler EF, Oppenheim RW, von Bartheld CS (1999) Neurotrophic factor regulation of developing avian oculomotor neurons: differential effects of BNDF and GDNF. J Neurobiol 41:295-315.

Sumner BEH (1975) A quantitative analysis of boutons with different types of synapse in normal and injured hypoglossal nuclei. Exp Neurol 49:406-417.

Sumner BEH (1976) Quantitative ultrastructural observations of the inhibited recovery of the hypoglossal nucleus from the axotomy response when regeneration of the hypoglossal nerve is prevented. Exp Brain Res 26:141-150.

Tapley P, Lamballe F, Barbacid M (1992) K252a is a selective inhibitor of the tyrosine protein kinase activity of the Trk family of oncogenes and neurotrophin receptors. Oncogene 7:371-381.

Titmus MJ, Faber DS (1990) Axotomy-induced alterations in the electrophysiological characteristics of neurons. Prog Neurobiol 35:1-51.

Ugolini G, Klam F, Doldan Dans M, Dubayle D, Brandi AM, Büttner-Ennever J, Graf W (2006) Horizontal eye movement networks in primates as revealed by retrograde transneuronal transfer of rabies virus: differences in monosynaptic input to "slow" and "fast" abducens motoneurons. J Comp Neurol 498:762-785.

Vanden Noven S, Pinter MJ (1989) Effects of preventing reinnervation on axotomized spinal motoneurons in the cat. II. Changes in group Ia synaptic function. J Neurophysiol 62:325-333.

Vicario-Abejón C, Owens D, McKay R, Segal M (2002) Role of neurotrophins in central synapse formation and stabilization. Nat Rev Neurosci 3:965-974.

Youssoufian M, Walmsley B (2007) Brain-derived neurotrophic factor modulates cells stability in the mouse medial nucleus of the trapezoid body. Eur J Neurosci 25:1647-1652.

Zhou Z, Liu Q, Davis RL (2005) Complex regulation of spiral ganglion neuron firing patterns by neurotrophin-3. J Neurosci 25:7558-7566.

Zweifel LS, Kuruvilla R, Ginty DD (2005) Functions and mechanisms of retrograde neurotrophin signaling. Nat Rev Neurosci 6:615-625. 